\title{
On Some Common Fixed Point Results for Weakly Contraction Mappings with Application
}

\author{
Yan Hao $(i)$ and Hongyan Guan (iD) \\ School of Mathematics and Systems Science, Shenyang Normal University, Shenyang 110034, China \\ Correspondence should be addressed to Hongyan Guan; guanhy8010@163.com
}

Received 3 February 2021; Revised 1 March 2021; Accepted 12 March 2021; Published 5 April 2021

Academic Editor: Huseyin Isik

Copyright ( 2021 Yan Hao and Hongyan Guan. This is an open access article distributed under the Creative Commons Attribution License, which permits unrestricted use, distribution, and reproduction in any medium, provided the original work is properly cited.

In this paper, we introduce a new class of generalized weakly contractive mappings and prove common fixed point results by using different algorithms involving this new class of mappings in the framework of $b$-metric spaces, which generalize the results of Cho. We also provide two examples to show the applicability and validity of our results. As an application of our result, we obtain a solution to an integral equation. Our results extend and improve several comparable results in the existing literature.

\section{Introduction}

The Banach fixed point theorem [1] popularly known as the Banach contraction mapping principle is a rewarding result in fixed point theory. It has widespread applications in both pure and applied mathematics and has been extended in many different directions. One of the most popular and interesting topics among them is the study of new classes of spaces and their fundamental properties.

In 1993, Czerwik [2] introduced firstly the concept of $b$ -metric space and proved some fixed point theorems of contractive mappings in $b$-metric space. After that, some authors have researched on the fixed point theorems of various new types of contractive conditions in $b$-metric space. Aydi et al. in [3] proved common fixed point results for single-valued and multivalued mappings satisfying a weak $\phi$-contraction in $b$-metric spaces. Starting from the results of Berinde [4], Pacurar [5] proved the existence and uniqueness of the fixed point of $\phi$-contractions and Zada et al. [6] established fixed point results satisfying contractive conditions of rational type. In 2019, Hussain et al. studied the existence and uniqueness of periodic common fixed point for pairs of mappings via rational type contraction in [7]. After that, in [8], the authors obtained fixed point theorems for cyclic $(\alpha, \beta)$ $-(\psi, \phi)_{s}$-rational type contractions and discussed the existence of a unique solution to nonlinear fractional differential equations. Also using rational type contractive conditions, Hussain et al. [9] got the existence and uniqueness of a common $n$-tupled fixed point for a pair of mappings. Using a contraction condition defined by means of a comparison function, [10] established results regarding the common fixed points of two mappings. In 2014, Abbas et al. obtained the results on common fixed point of four mappings in $b$ -metric space in [11]. Iqbal et al. [12] introduced a generalized multivalued $(\alpha, L)$-almost contraction and proved the existence and uniqueness of the fixed point for a specific mapping in the $b$-metric space.

Inspired by Czerwik's results, Hussain and Shah in [13] introduced the notion of a cone $b$-metric space, which means that it is a generalization of $b$-metric spaces and cone metric spaces; they considered topological properties of cone $b$ -metric spaces and obtained some results on KKM mappings in the setting of cone $b$-metric spaces. Younis et al. [14] studied the existence of fixed points of a new class of generalized $F$-contraction in partial $b$-metric space. In [15], some fixed point results for weakly contractive mappings in ordered partial metric space were obtained. Recently, Samet et al. [16] introduced the concept of $\alpha$-admissible and $\alpha-\psi$-contractive mappings and presented fixed point theorems for them. In $[17,18]$, Zoto et al. studied generalized $\alpha_{s^{p}}$ contractive mappings and $(\alpha-\psi, \phi)$-contractions in $b$-metric-like space. In 2020, Isik et al. [19] firstly introduced the structure of 
extended quasi $b$-metric-like spaces as a generalization of both quasi metric-like spaces and quasi $b$-metric-like spaces. Also, they presented the notion of JSR-contractive mappings in the setup of extended quasi $b$-metric-like spaces and investigated the existence of fixed point for such mappings. AbuDonia et al. [20] proved the uniqueness and existence of the fixed points for five mappings from a complete intuitionistic fuzzy 3-metric space into itself under weak compatible of type $(\alpha)$ and asymptotically regular. In 2015, Ege [21] introduced complex valued rectangular $b$-metric space and proved an analogue of the Banach contraction principle in this space. Recently, Younis et al. [22] provided much simpler and shorter proofs of some new results in rectangular metric spaces, and Mitrovic et al. [23] gave a proof of the results of Miculescu and Mihail [24] and Suzuki [25] in extended $b$ -metric spaces. In graphical $b$-metric spaces, Younis et al. presented fixed point results for Kannan-type and Reichtype mappings in [26, 27]. Lately, Gholidahneh et al. [28] introduced the notion of a modular $p$-metric space (an extended modular $b$-metric space) and established some fixed point results for $\alpha-\widehat{v}$-Meir-Keeler contractions in this new space.

In 1997, Alber and Guerre-Delabriere [29] generalized the Banach fixed point theorem by introducing the concept of weak contraction mappings in Hilbert spaces. Weak contraction principle states that every weak contraction mapping on a complete Hilbert space has a unique fixed point. Rhoades [30] extended weak contraction principle in Hilbert spaces to metric spaces. Since then, many authors (for example, [31-42]) obtained generalizations and extensions of the weak contraction principle. Recently, in [43], Jamal et al. used $(\psi, \phi)$-weak contraction to generalize coincidence point results which are established in the context of partially ordered $b$-metric spaces.

In particular, Choudhury et al. [36] obtained a generalization of the weak contraction principle in metric spaces by using altering distance functions as follows:

Theorem 1 (see [36]). Suppose that a mapping $g: X \longrightarrow X$, where $X$ is a metric space with metric $d$, satisfies the following condition:

$$
\begin{aligned}
\psi(d(g x, g y)) \leq & \psi\left(\max \left\{d(x, y), d(x, g x), d(y, g y), \frac{1}{2}\{d(x, g y)+d(y, g x)\}\right\}\right) \\
& -\varphi(\max \{d(x, y), d(y, g y)\}),
\end{aligned}
$$

for all $x, y \in X$, where $\varphi:[0,+\infty) \longrightarrow[0,+\infty)$ is a continuous function and $\psi:[0,+\infty) \longrightarrow[0,+\infty)$ is an altering function, that is, $\psi$ is a nondecreasing and continuous function, and $\psi$ $(t)=0$ if and only if $t=0$. Then, $g$ has a unique fixed point.

Let $X$ be a metric space with metric $d$, let $T: X \longrightarrow X$, and let $\varphi: X \longrightarrow[0,+\infty)$ be a lower semicontinuous function. Then, $T$ is called a generalized weakly contractive mapping if it satisfies the following condition:

$$
\begin{aligned}
& \psi(d(T x, T y)+\varphi(T x)+\varphi(T y)) \\
& \quad \leq \psi(m(x, y, d, T, \varphi))-\phi(l(x, y, d, T, \varphi)),
\end{aligned}
$$

where $\psi \in \Psi, \phi \in \Phi$, and

$$
\begin{aligned}
m(x, y, d, T, \varphi)= & \max \{d(x, y)+\varphi(x)+\varphi(y), d(x, T x)+\varphi(x) \\
& +\varphi(T x), d(y, T y)+\varphi(y)+\varphi(T y), \frac{1}{2}\{d(x, T y) \\
& +\varphi(x)+\varphi(T y)+d(y, T x)+\varphi(y)+\varphi(T x)\}\},
\end{aligned}
$$

$l(x, y, d, T, \varphi)=\max \{d(x, y)+\varphi(x)+\varphi(y), d(y, T y)+\varphi(y)+\varphi(T y)\}$.

Cho [44] extended the results of Choudhury et al. [36] to generalized weakly contractive mappings in the setting of metric spaces and obtained the following result:

Theorem 2 (see [44]). Let $X$ be complete. If $T$ is a generalized weakly contractive mapping, then there exists a unique $z \in X$ such that $z=T z$ and $\varphi(z)=0$.

Motivated and inspired by Theorem 2.1 in [44], in this paper, our purpose is to introduce a new class of generalized weakly contractive mappings and obtain a few of common fixed point results by using different algorithms involving generalized weakly contractive conditions in the framework of $b$-metric space, which generalize the results of Cho. Furthermore, we provide examples that elaborated the useability of our results. Meanwhile, we present an application to the existence of solutions to an integral equation by means of one of our results.

\section{Preliminaries}

In this section, in order to get our main results, we will introduce some definitions and lemmas first.

Definition 3 (see [2]). Let $X$ be a nonempty set and $s \geq 1$ be a given real number. A mapping $d: X \times X \longrightarrow[0,+\infty)$ is said to be a $b$-metric if and only if, for all $x, y, z \in X$, the following conditions are satisfied:

(i) $d(x, y)=0$ if and only if $x=y$,

(ii) $d(x, y)=d(y, x)$,

(iii) $d(x, y) \leq s(d(x, z)+d(y, z))$.

In general, $(X, d)$ is called a $b$-metric space with parameter $s \geq 1$.

Remark 4 . We should note that a $b$-metric space with $s=1$ is a metric space. We can find several examples of $b$-metric spaces which are not metric spaces (see [45]). 
Example 5 (see [46]). Let $(X, \rho)$ be a metric space, and $d(x$, $y)=(\rho(x, y))^{p}$, where $p>1$ is a real number. Then, $d(x, y)$ is a $b$-metric space with $s=2^{p-1}$.

Definition 6 (see [11]). Let $(X, d)$ be a $b$-metric space with parameter $s \geq 1$. Then, a sequence $\left\{x_{n}\right\}$ in $X$ is said to be

(i) $b$-convergent if and only if there exists $x \in X$ such that $d\left(x_{n}, x\right) \longrightarrow 0$ as $n \longrightarrow+\infty$,

(ii) a Cauchy sequence if and only if $d\left(x_{n}, x_{m}\right) \longrightarrow 0$ when $n, m \longrightarrow+\infty$.

In addition, a $b$-metric space is called complete if and only if each Cauchy sequence in this space is $b$-convergent.

Definition 7 (see [47]). Let $f$ and $g$ be two self-mappings on a nonempty set $X$. If $w=f x=g x$, for some $x \in X$, then $x$ is said to be the coincidence point of $f$ and $g$, where $w$ is called the point of coincidence of $f$ and $g$. Let $C(f, g)$ denote the set of all coincidence points of $f$ and $g$.

Definition 8 (see [47]). Let $f$ and $g$ be two self-mappings defined on a nonempty set $X$. Then, $f$ and $g$ is said to be weakly compatible if they commute at every coincidence point, that is, $f x=g x \Rightarrow f g x=g f x$ for every $x \in C(f, g)$.

The following lemma plays an important role to obtain our main results:

Lemma 9 (see [46]). Let $(X, d)$ be a $b$-metric space with parameter $s \geq 1$. Assume that $\left\{x_{n}\right\}$ and $\left\{y_{n}\right\}$ are $b$-convergent to $x$ and $y$, respectively. Then, we have

$$
\frac{1}{s^{2}} d(x, y) \leq \liminf _{n \longrightarrow+\infty} d\left(x_{n}, y_{n}\right) \leq \limsup _{n \longrightarrow+\infty} d\left(x_{n}, y_{n}\right) \leq s^{2} d(x, y)
$$

In particular, if $x=y$, then we have $\lim _{n \longrightarrow+\infty} d\left(x_{n}, y_{n}\right)$ $=0$. Moreover, for each $z \in X$, we have

$$
\frac{1}{s} d(x, z) \leq \liminf _{n \longrightarrow+\infty} d\left(x_{n}, z\right) \leq \limsup _{n \longrightarrow+\infty} d\left(x_{n}, z\right) \leq s d(x, z)
$$

\section{Main Results}

In this section, we will establish common fixed point theorems for generalized weakly contractive mappings in complete $b$-metric space. Furthermore, we also provide two examples to support our results.

A function $f: X \longrightarrow[0,+\infty)$, where $(X, d)$ is a $b$-metric space, is called lower semicontinuous if, for all $x \in X$ and $\left\{x_{n}\right\}$ are $b$-convergent to $x$, we have

$$
f(x) \leq \liminf _{n \longrightarrow+\infty} f\left(x_{n}\right)
$$

We shall consider that the contractive conditions in this section are constructed via auxiliary functions defined with the families $\Psi, \Phi$, respectively:

$$
\Psi=\{\psi:[0,+\infty) \rightarrow[0,+\infty) \text { is a nondecreasing and continuous function }\},
$$

$$
\begin{aligned}
\Phi & =\{\phi:[0,+\infty) \\
& \longrightarrow[0,+\infty) \text { is a nondecreasing and lower semicontinuous function and } \phi(t) \\
& =0 \text { if and only if } t=0\} .
\end{aligned}
$$

Theorem 10. Let $(X, d)$ be a complete b-metric space with parameter $s \geq 1$, and let $f, g: X \longrightarrow X$ be given selfmappings satisfying $g$ as injective and $f(X) \subset g(X)$ where $g(X)$ is closed. Suppose $\varphi: X \longrightarrow[0,+\infty)$ is a lower semicontinuous function and $p \geq 2$ is a constant. If there are functions $\psi \in \Psi$ and $\phi \in \Phi$ such that

$$
\begin{aligned}
& \psi\left(s^{p}[d(f x, f y)+\varphi(f x)+\varphi(f y)]\right) \\
& \quad \leq \psi(m(x, y, d, f, g, \varphi))-\phi(l(x, y, d, f, g, \varphi)),
\end{aligned}
$$

where

$$
\begin{aligned}
m(x, y, d, f, g, \varphi)= & \max \left\{d(g x, g y)+\varphi(g x)+\varphi(g y), \frac{1}{2}\{d(f x, g x)+\varphi(f x)\right. \\
& +\varphi(g x)+d(f y, g y)+\varphi(f y)+\varphi(g y)\}, \frac{1}{2 s}\{d(f x, g y) \\
& +\varphi(f x)+\varphi(g y)+d(f y, g x)+\varphi(f y)+\varphi(g x)\}\}
\end{aligned}
$$

$l(x, y, d, f, g, \varphi)=\max \{d(g x, g y)+\varphi(g x)+\varphi(g y), d(f y, g y)+\varphi(f y)+\varphi(g y)\}$,

then $f$ and $g$ have a unique coincidence point in X. Moreover, $f$ and $g$ have a unique common fixed point provided that $f$ and $g$ are weakly compatible.

Proof. Let $x_{0} \in X$. As $f(X) \subset g(X)$, there exists $x_{1} \in X$ with $f$ $x_{0}=g x_{1}$. Now we define the sequences $\left\{x_{n}\right\}$ and $\left\{y_{n}\right\}$ in $X$ by $y_{n}=f x_{n}=g x_{n+1}$ for all $n \in \mathbb{N}$. If $y_{n}=y_{n+1}$ for some $n \in$ $\mathbb{N}$; then, we have $y_{n}=y_{n+1}=f x_{n+1}=g x_{n+1}$ and $f$ and $g$ have a coincidence point. Without loss of generality, we assume that $y_{n} \neq y_{n+1}$ for all $n \in \mathbb{N}$. Applying (10) with $x=x_{n}$ and $y$ $=x_{n+1}$, we obtain

$$
\begin{aligned}
& \psi\left(d\left(y_{n}, y_{n+1}\right)+\varphi\left(y_{n}\right)+\varphi\left(y_{n+1}\right)\right) \\
& \quad \leq \psi\left(s^{p}\left[d\left(y_{n}, y_{n+1}\right)+\varphi\left(y_{n}\right)+\varphi\left(y_{n+1}\right)\right]\right) \\
& \quad=\psi\left(s^{p}\left[d\left(f x_{n}, f x_{n+1}\right)+\varphi\left(f x_{n}\right)+\varphi\left(f x_{n+1}\right)\right]\right) \\
& \quad \leq \psi\left(m\left(x_{n}, x_{n+1}, d, f, g, \varphi\right)\right)-\phi\left(l\left(x_{n}, x_{n+1}, d, f, g, \varphi\right)\right),
\end{aligned}
$$


where

$$
\begin{aligned}
m\left(x_{n}, x_{n+1}, d, f, g, \varphi\right)= & \max \left\{d\left(g x_{n}, g x_{n+1}\right)+\varphi\left(g x_{n}\right)\right. \\
& +\varphi\left(g x_{n+1}\right), \frac{1}{2}\left\{d\left(f x_{n}, g x_{n}\right)+\varphi\left(f x_{n}\right)+\varphi\left(g x_{n}\right)\right. \\
& +d\left(f x_{n+1}, g x_{n+1}\right)+\varphi\left(f x_{n+1}\right) \\
& \left.+\varphi\left(g x_{n+1}\right)\right\}, \frac{1}{2 s}\left\{d\left(f x_{n}, g x_{n+1}\right)+\varphi\left(f x_{n}\right)+\varphi\left(g x_{n+1}\right)\right. \\
& \left.\left.+d\left(f x_{n+1}, g x_{n}\right)+\varphi\left(f x_{n+1}\right)+\varphi\left(g x_{n}\right)\right\}\right\} \\
\leq & \max \left\{d\left(y_{n-1}, y_{n}\right)+\varphi\left(y_{n-1}\right)+\varphi\left(y_{n}\right), \frac{1}{2}\left\{d\left(y_{n}, y_{n-1}\right)\right.\right. \\
& +\varphi\left(y_{n}\right)+\varphi\left(y_{n-1}\right)+d\left(y_{n+1}, y_{n}\right)+\varphi\left(y_{n+1}\right) \\
& \left.+\varphi\left(y_{n}\right)\right\}, \frac{1}{2 s}\left\{d\left(y_{n}, y_{n}\right)+\varphi\left(y_{n}\right)+\varphi\left(y_{n}\right)\right. \\
& \left.\left.+d\left(y_{n+1}, y_{n-1}\right)+\varphi\left(y_{n+1}\right)+\varphi\left(y_{n-1}\right)\right\}\right\} \\
& \leq \max \left\{d\left(y_{n-1}, y_{n}\right)+\varphi\left(y_{n-1}\right)+\varphi\left(y_{n}\right), d\left(y_{n+1}, y_{n}\right)\right. \\
& \left.+\varphi\left(y_{n+1}\right)+\varphi\left(y_{n}\right)\right\},
\end{aligned}
$$

$$
\begin{aligned}
l\left(x_{n}, x_{n+1}, d, f, g, \varphi\right)= & \max \left\{d\left(g x_{n}, g x_{n+1}\right)+\varphi\left(g x_{n}\right)+\varphi\left(g x_{n+1}\right), d\left(f x_{n+1}, g x_{n+1}\right)\right. \\
& \left.+\varphi\left(f x_{n+1}\right)+\varphi\left(g x_{n+1}\right)\right\}=\max \left\{d\left(y_{n-1}, y_{n}\right)+\varphi\left(y_{n-1}\right)\right. \\
& \left.+\varphi\left(y_{n}\right), d\left(y_{n+1}, y_{n}\right)+\varphi\left(y_{n+1}\right)+\varphi\left(y_{n}\right)\right\} .
\end{aligned}
$$

If $d\left(y_{n}, y_{n+1}\right)+\varphi\left(y_{n}\right)+\varphi\left(y_{n+1}\right)>d\left(y_{n}, y_{n-1}\right)+\varphi\left(y_{n}\right)+\varphi$ $\left(y_{n-1}\right)$, for some $n \in \mathbb{N}$, in view of (12), (13), and (14), we have

$$
\begin{aligned}
\psi( & \left.d\left(y_{n}, y_{n+1}\right)+\varphi\left(y_{n}\right)+\varphi\left(y_{n+1}\right)\right) \\
\leq & \psi\left(m\left(x_{n}, x_{n+1}, d, f, g, \varphi\right)\right)-\phi\left(l\left(x_{n}, x_{n+1}, d, f, g, \varphi\right)\right) \\
\leq & \psi\left(d\left(y_{n+1}, y_{n}\right)+\varphi\left(y_{n+1}\right)+\varphi\left(y_{n}\right)\right) \\
\quad & -\phi\left(d\left(y_{n+1}, y_{n}\right)+\varphi\left(y_{n+1}\right)+\varphi\left(y_{n}\right)\right),
\end{aligned}
$$

which implies $\phi\left(d\left(y_{n}, y_{n+1}\right)+\varphi\left(y_{n}\right)+\varphi\left(y_{n+1}\right)\right)=0$. Hence, $y_{n}=y_{n+1}$, a contradiction.

Thus, we have

$$
d\left(y_{n}, y_{n+1}\right)+\varphi\left(y_{n}\right)+\varphi\left(y_{n+1}\right) \leq d\left(y_{n}, y_{n-1}\right)+\varphi\left(y_{n}\right)+\varphi\left(y_{n-1}\right),
$$

$$
\begin{gathered}
m\left(x_{n}, x_{n+1}, d, f, g, \varphi\right) \leq d\left(y_{n}, y_{n-1}\right)+\varphi\left(y_{n}\right)+\varphi\left(y_{n-1}\right), \\
l\left(x_{n}, x_{n+1}, d, f, g, \varphi\right)=d\left(y_{n}, y_{n-1}\right)+\varphi\left(y_{n}\right)+\varphi\left(y_{n-1}\right) .
\end{gathered}
$$

It follows from (16) that $\left\{d\left(y_{n}, y_{n+1}\right)+\varphi\left(y_{n}\right)+\varphi\left(y_{n+1}\right)\right\}$ is a nonincreasing sequence, and so there exists $r \geq 0$ such that

$$
\lim _{n \longrightarrow+\infty}\left(d\left(y_{n}, y_{n+1}\right)+\varphi\left(y_{n}\right)+\varphi\left(y_{n+1}\right)\right)=r .
$$

By virtue of (12), (17), and (18), one can obtain

$$
\begin{aligned}
\psi( & \left.d\left(y_{n}, y_{n+1}\right)+\varphi\left(y_{n}\right)+\varphi\left(y_{n+1}\right)\right) \\
\leq & \psi\left(m\left(x_{n}, x_{n+1}, d, f, g, \varphi\right)\right)-\phi\left(l\left(x_{n}, x_{n+1}, d, f, g, \varphi\right)\right) \\
\leq & \psi\left(d\left(y_{n}, y_{n-1}\right)+\varphi\left(y_{n}\right)+\varphi\left(y_{n-1}\right)\right) \\
& \quad-\phi\left(d\left(y_{n}, y_{n-1}\right)+\varphi\left(y_{n}\right)+\varphi\left(y_{n-1}\right)\right) .
\end{aligned}
$$

Now assume that $r>0$. Taking the upper limit as $n \longrightarrow$ $\infty$ in (20), we have

$$
\begin{aligned}
& \limsup _{n \longrightarrow+\infty} \psi\left(d\left(y_{n}, y_{n+1}\right)+\varphi\left(y_{n}\right)+\varphi\left(y_{n+1}\right)\right) \\
& \leq \limsup _{n \longrightarrow+\infty} \psi\left(m\left(x_{n}, x_{n+1}, d, f, g, \varphi\right)\right) \\
& \quad-\limsup _{n \longrightarrow+\infty} \phi\left(l\left(x_{n}, x_{n+1}, d, f, g, \varphi\right)\right) \\
& \leq \limsup \psi\left(d\left(y_{n}, y_{n-1}\right)+\varphi\left(y_{n}\right)+\varphi\left(y_{n-1}\right)\right) \\
& \quad-\liminf _{n \longrightarrow+\infty} \phi\left(d\left(y_{n}, y_{n-1}\right)+\varphi\left(y_{n}\right)+\varphi\left(y_{n-1}\right)\right),
\end{aligned}
$$

which implies that $\psi(r) \leq \psi(r)-\phi(r)$, a contradiction. This yields that

$$
\lim _{n \longrightarrow+\infty}\left(d\left(y_{n}, y_{n+1}\right)+\varphi\left(y_{n}\right)+\varphi\left(y_{n+1}\right)\right)=r=0 .
$$

It follows that $\lim _{n \longrightarrow+\infty} d\left(y_{n}, y_{n+1}\right)=0$ and $\lim _{n \longrightarrow+\infty} \varphi($ $\left.y_{n}\right)=0$.

Now we shall prove that $\left\{y_{n}\right\}$ is a Cauchy sequence in $X$. Suppose on the contrary that $\left\{y_{n}\right\}$ is not Cauchy. It follows that there exists $\varepsilon>0$ for which one can find sequences \{ $\left.y_{m_{k}}\right\}$ and $\left\{y_{n_{k}}\right\}$ of $\left\{y_{n}\right\}$ satisfying $n_{k}$ is the smallest index for which $n_{k}>m_{k}>k$,

$$
\begin{gathered}
\varepsilon \leq d\left(y_{m_{k}}, y_{n_{k}}\right), \\
d\left(y_{m_{k}}, y_{n_{k}-1}\right)<\varepsilon .
\end{gathered}
$$

By the triangle inequality in $b$-metric space and (23) and (24), we have

$$
\begin{aligned}
\varepsilon & \leq d\left(y_{m_{k}}, y_{n_{k}}\right) \leq s d\left(y_{m_{k}}, y_{n_{k}-1}\right)+s d\left(y_{n_{k}-1}, y_{n_{k}}\right) \\
& <s \varepsilon+s d\left(y_{n_{k}-1}, y_{n_{k}}\right) .
\end{aligned}
$$

Taking the upper limit as $k \longrightarrow+\infty$ in the above inequality, we have

$$
\varepsilon \leq \limsup _{k \longrightarrow+\infty} d\left(y_{m_{k}}, y_{n_{k}}\right) \leq s \varepsilon
$$

Also,

$$
d\left(y_{m_{k}}, y_{n_{k}}\right) \leq s d\left(y_{m_{k}}, y_{n_{k}-1}\right)+s d\left(y_{n_{k}-1}, y_{n_{k}}\right)
$$




$$
\begin{gathered}
d\left(y_{m_{k}}, y_{n_{k}}\right) \leq s d\left(y_{m_{k}}, y_{m_{k}-1}\right)+s d\left(y_{m_{k}-1}, y_{n_{k}}\right), \\
d\left(y_{m_{k}-1}, y_{n_{k}}\right) \leq s d\left(y_{m_{k}-1}, y_{m_{k}}\right)+s d\left(y_{m_{k}}, y_{n_{k}}\right) .
\end{gathered}
$$

From (23), (24), and (27), we obtain

$$
\frac{\varepsilon}{s} \leq \limsup _{k \longrightarrow+\infty} d\left(y_{m_{k}}, y_{n_{k}-1}\right) \leq \varepsilon
$$

Using (23), (28), and (29), we get

$$
\frac{\varepsilon}{s} \leq \limsup _{k \longrightarrow+\infty} d\left(y_{m_{k}-1}, y_{n_{k}}\right) \leq s^{2} \varepsilon
$$

Similarly,

$$
\begin{aligned}
d\left(y_{m_{k}-1}, y_{n_{k}-1}\right) \leq & s d\left(y_{m_{k}-1}, y_{m_{k}}\right)+s d\left(y_{m_{k}}, y_{n_{k}-1}\right) \\
d\left(y_{m_{k}}, y_{n_{k}}\right) \leq & s d\left(y_{m_{k}}, y_{m_{k}-1}\right)+s^{2} d\left(y_{m_{k}-1}, y_{n_{k}-1}\right) \\
& +s^{2} d\left(y_{n_{k}-1}, y_{n_{k}}\right)
\end{aligned}
$$

so there is

$$
\frac{\varepsilon}{s^{2}} \leq \limsup _{k \longrightarrow+\infty} d\left(y_{m_{k}-1}, y_{n_{k}-1}\right) \leq s \varepsilon
$$

Using the same method, one can obtain that

$$
\begin{gathered}
\quad \varepsilon \leq \liminf _{k \longrightarrow+\infty} d\left(y_{m_{k}}, y_{n_{k}}\right) \leq s \mathcal{E}, \frac{\varepsilon}{s} \leq \liminf _{k \longrightarrow+\infty} d\left(y_{m_{k}}, y_{n_{k}-1}\right) \leq \varepsilon, \\
\frac{\varepsilon}{s} \leq \liminf _{k \longrightarrow+\infty} d\left(y_{m_{k}-1}, y_{n_{k}}\right) \leq s^{2} \varepsilon, \frac{\varepsilon}{s^{2}} \leq \liminf _{k \longrightarrow+\infty} d\left(y_{m_{k}-1}, y_{n_{k}-1}\right) \leq s \varepsilon .
\end{gathered}
$$

In view of the definition of $m(x, y, d, f, g, \varphi)$, we deduce

$$
\begin{aligned}
m\left(x_{m_{k}}, x_{n_{k}}, d, f, g, \varphi\right)= & \max \left\{d\left(g x_{m_{k}}, g x_{n_{k}}\right)+\varphi\left(g x_{m_{k}}\right)+\varphi\left(g x_{n_{k}}\right), \frac{1}{2}\left\{d\left(f x_{m_{k}}, g x_{m_{k}}\right)\right.\right. \\
& +\varphi\left(f x_{m_{k}}\right)+\varphi\left(g x_{m_{k}}\right)+d\left(f x_{n_{k}}, g x_{n_{k}}\right)+\varphi\left(f x_{n_{k}}\right) \\
& \left.+\varphi\left(g x_{n_{k}}\right)\right\}, \frac{1}{2 s}\left\{d\left(f x_{m_{k}}, g x_{n_{k}}\right)+\varphi\left(f x_{m_{k}}\right)+\varphi\left(g x_{n_{k}}\right)\right. \\
& \left.\left.+d\left(f x_{n_{k}}, g x_{m_{k}}\right)+\varphi\left(f x_{n_{k}}\right)+\varphi\left(g x_{m_{k}}\right)\right\}\right\} \\
= & \max \left\{d\left(y_{m_{k}-1}, y_{n_{k}-1}\right)+\varphi\left(y_{m_{k}-1}\right)+\varphi\left(y_{n_{k}-1}\right), \frac{1}{2}\right. \\
& +\left\{d\left(y_{m_{k}}, y_{m_{k}-1}\right)+\varphi\left(y_{m_{k}}\right)+\varphi\left(y_{m_{k}-1}\right)+d\left(y_{n_{k}}, y_{n_{k}-1}\right)\right. \\
& \left.+\varphi\left(y_{n_{k}}\right)+\varphi\left(y_{n_{k}-1}\right)\right\}, \frac{1}{2 s}\left\{d\left(y_{m_{k}}, y_{n_{k}-1}\right)+\varphi\left(y_{m_{k}}\right)\right. \\
& \left.\left.+\varphi\left(y_{n_{k}-1}\right)+d\left(y_{n_{k}}, y_{m_{k}-1}\right)+\varphi\left(y_{n_{k}}\right)+\varphi\left(y_{m_{k}-1}\right)\right\}\right\} .
\end{aligned}
$$

Taking the upper limit as $k \longrightarrow+\infty$ in (35), we obtain

$$
\limsup _{k \longrightarrow+\infty} m\left(x_{m_{k}}, x_{n_{k}}, d, f, g, \varphi\right) \leq \max \left\{s \varepsilon, 0, \frac{\varepsilon+s^{2} \varepsilon}{2 s}\right\}=s \varepsilon
$$

Also, we have

$$
\begin{aligned}
l\left(x_{m_{k}}, x_{n_{k}}, d, f, g, \varphi\right)= & \max \left\{d\left(g x_{m_{k}}, g x_{n_{k}}\right)+\varphi\left(g x_{m_{k}}\right)+\varphi\left(g x_{n_{k}}\right), d\left(f x_{n_{k}}, g x_{n_{k}}\right)\right. \\
& \left.+\varphi\left(f x_{n_{k}}\right)+\varphi\left(g x_{n_{k}}\right)\right\} \\
= & \max \left\{d\left(y_{m_{k}-1}, y_{n_{k}-1}\right)+\varphi\left(y_{m_{k}-1}\right)+\varphi\left(y_{n_{k}-1}\right), d\left(y_{n_{k}}, y_{n_{k}-1}\right)\right. \\
& \left.+\varphi\left(y_{n_{k}}\right)+\varphi\left(y_{n_{k}-1}\right)\right\} .
\end{aligned}
$$

It follows that

$$
s \varepsilon \geq \liminf _{k \longrightarrow+\infty} l\left(x_{m_{k}}, x_{n_{k}}, d, f, g, \varphi\right) \geq \frac{\varepsilon}{s^{2}}
$$

Applying (10) with $x=x_{m_{k}}$ and $y=x_{n_{k}}$, one can get

$$
\begin{aligned}
\psi(s \mathcal{\varepsilon}) \leq & \psi\left(s^{p} \mathcal{\varepsilon}\right) \leq \psi\left(s^{p} \limsup _{n \longrightarrow+\infty}\left[d\left(y_{m_{k}}, y_{n_{k}}\right)+\varphi\left(y_{m_{k}}\right)+\varphi\left(y_{n_{k}}\right)\right]\right) \\
\leq & \psi\left(\limsup _{n \longrightarrow+\infty}\left(x_{m_{k}}, x_{n_{k}}, d, f, g, \varphi\right)\right) \\
& \quad-\liminf _{n \longrightarrow+\infty} \phi\left(l\left(x_{m_{k}}, x_{n_{k}}, d, f, g, \varphi\right)\right) \\
\leq & \psi(s \varepsilon)-\phi\left(\liminf _{n \longrightarrow+\infty} l\left(x_{m_{k}}, x_{n_{k}}, d, f, g, \varphi\right)\right),
\end{aligned}
$$

which implies that

$$
\liminf _{n \longrightarrow+\infty} l\left(x_{m_{k}}, x_{n_{k}}, d, f, g, \varphi\right)=0,
$$

a contradiction to (38). It follows that $\left\{y_{n}\right\}$ is a Cauchy sequence in $X$. The completeness of $X$ ensures that there exists a $u \in X$ such that

$$
\begin{aligned}
\lim _{n \longrightarrow+\infty} d\left(y_{n}, u\right) & =\lim _{n \longrightarrow+\infty} d\left(f x_{n}, u\right)=\lim _{n \longrightarrow+\infty} d\left(g x_{n+1}, u\right) \\
& =\lim _{n, m \longrightarrow+\infty} d\left(y_{n}, y_{m}\right)=0
\end{aligned}
$$

Furthermore, we have $u \in g(X)$ since $g(X)$ is closed. It follows that one can choose a $z \in X$ such that $u=g z$, and one can write (41) as

$$
\lim _{n \longrightarrow+\infty} d\left(y_{n}, g z\right)=\lim _{n \longrightarrow+\infty} d\left(f x_{n}, g z\right)=\lim _{n \longrightarrow+\infty} d\left(g x_{n+1}, g z\right)=0 .
$$

Following from the definition of $\varphi$, we get

$$
\varphi(g z)=\varphi(u) \leq \liminf _{n \longrightarrow+\infty} \varphi\left(y_{n}\right)=0
$$

That is, $\varphi(g z)=\varphi(u)=0$. 
If $f z \neq g z$, taking $x=x_{n_{k}}$ and $y=z$ in contractive condition (10), we deduce that

$$
\begin{aligned}
& \psi\left(d\left(f x_{n_{k}}, f z\right)+\varphi\left(f x_{n_{k}}\right)+\varphi(f z)\right) \\
& \quad \leq \psi\left(s^{p}\left[d\left(f x_{n_{k}}, f z\right)+\varphi\left(f x_{n_{k}}\right)+\varphi(f z)\right]\right) \\
& \quad \leq \psi\left(m\left(x_{n_{k}}, z, d, f, g, \varphi\right)\right)-\phi\left(l\left(x_{n_{k}}, z, d, f, g, \varphi\right)\right),
\end{aligned}
$$

where

$$
\begin{aligned}
m\left(x_{n_{k}}, z, d, f, g, \varphi\right)= & \max \left\{d\left(g x_{n_{k}}, g z\right)+\varphi\left(g x_{n_{k}}\right)+\varphi(g z), \frac{1}{2}\left\{d\left(f x_{n_{k}}, g x_{n_{k}}\right)\right.\right. \\
& \left.+\varphi\left(f x_{n_{k}}\right)+\varphi\left(g x_{n_{k}}\right)+d(f z, g z)+\varphi(f z)+\varphi(g z)\right\}, \frac{1}{2 s} \\
& \cdot\left\{d\left(f x_{n_{k}}, g z\right)+\varphi\left(f x_{n_{k}}\right)+\varphi(g z)+d\left(f z, g x_{n_{k}}\right)+\varphi(f z)\right. \\
& \left.\left.+\varphi\left(g x_{n_{k}}\right)\right\}\right\}=\max \left\{d\left(y_{n_{k}-1}, g z\right)+\varphi\left(y_{n_{k}-1}\right)+\varphi(g z), \frac{1}{2}\right. \\
& \cdot\left\{d\left(y_{n_{k}}, y_{n_{k}-1}\right)+\varphi\left(y_{n_{k}}\right)+\varphi\left(y_{n_{k}-1}\right)+d(f z, g z)+\varphi(f z)\right. \\
& +\varphi(g z)\}, \frac{1}{2 s}\left\{d\left(y_{n_{k}}, g z\right)+\varphi\left(y_{n_{k}}\right)+\varphi(g z)\right. \\
& \left.\left.+d\left(f z, y_{n_{k}-1}\right)+\varphi(f z)+\varphi\left(y_{n_{k}-1}\right)\right\}\right\}, \\
l\left(x_{n_{k}}, z, d, f, g, \varphi\right)= & \max \left\{d\left(g x_{n_{k}}, g z\right)+\varphi\left(g x_{n_{k}}\right)+\varphi(g z), d(f z, g z)\right. \\
& +\varphi(f z)+\varphi(g z)\}=\max \left\{d\left(y_{n_{k}-1}, g z\right)\right. \\
& \left.+\varphi\left(y_{n_{k}-1}\right)+\varphi(g z), d(f z, g z)+\varphi(f z)+\varphi(g z)\right\} .
\end{aligned}
$$

By simple calculation, we obtain

$$
\begin{aligned}
& \limsup _{k \longrightarrow+\infty} m\left(x_{n_{k}}, z, d, f, g, \varphi\right) \leq d(f z, g z)+\varphi(f z), \\
& \liminf _{k \longrightarrow+\infty} l\left(x_{n_{k}}, z, d, f, g, \varphi\right) \geq d(f z, g z)+\varphi(f z) .
\end{aligned}
$$

By taking the upper limit as $k \longrightarrow+\infty$ in (44) and using (46) and (47), one can get

$$
\begin{aligned}
\psi(d(f z, g z)+\varphi(f z)) \leq & \psi(d(f z, g z)+\varphi(f z)) \\
& -\phi(d(f z, g z)+\varphi(f z)) .
\end{aligned}
$$

Hence, $d(f z, g z)+\varphi(f z)=0$, which implies that $f z=g z$ and $\varphi(f z)=0$.

Now we claim that $z$ is the unique coincidence point of $f$ and $g$. If not, there exist $z, z^{\prime} \in C(f, g)$ and $z \neq z^{\prime}$; applying (10) with $x=z$ and $y=z^{\prime}$, we obtain that

$$
\begin{aligned}
& \psi\left(d\left(f z, f z^{\prime}\right)+\varphi(f z)+\varphi\left(f z^{\prime}\right)\right) \\
& \quad \leq \psi\left(s^{p}\left[d\left(f z, f z^{\prime}\right)+\varphi(f z)+\varphi\left(f z^{\prime}\right)\right]\right) \\
& \quad \leq \psi\left(m\left(z, z^{\prime}, d, f, g, \varphi\right)\right)-\phi\left(l\left(z, z^{\prime}, d, f, g, \varphi\right)\right) .
\end{aligned}
$$

Here,

$$
\begin{aligned}
m\left(z, z^{\prime}, d, f, g, \varphi\right)= & \max \left\{d\left(g z, g z^{\prime}\right)+\varphi(g z)+\varphi\left(g z^{\prime}\right), \frac{1}{2}\{d(f z, g z)\right. \\
& \left.+\varphi(f z)+\varphi(g z)+d\left(f z^{\prime}, g z^{\prime}\right)+\varphi\left(f z^{\prime}\right)+\varphi\left(g z^{\prime}\right)\right\}, \frac{1}{2 s} \\
& \cdot\left\{d\left(f z, g z^{\prime}\right)+\varphi(f z)+\varphi\left(g z^{\prime}\right)+d\left(f z^{\prime}, g z\right)+\varphi\left(f z^{\prime}\right)\right. \\
& +\varphi(g z)\}\} \leq d\left(g z, g z^{\prime}\right)+\varphi\left(g z^{\prime}\right), \\
l\left(z, z^{\prime}, d, f, g, \varphi\right)= & \max \left\{d\left(g z, g z^{\prime}\right)+\varphi(g z)+\varphi\left(g z^{\prime}\right), d\left(f z^{\prime}, g z^{\prime}\right)\right. \\
& \left.+\varphi\left(f z^{\prime}\right)+\varphi\left(g z^{\prime}\right)\right\} \geq d\left(g z, g z^{\prime}\right)+\varphi\left(g z^{\prime}\right) .
\end{aligned}
$$

It follows from (49) that

$\psi\left(d\left(g z, g z^{\prime}\right)+\varphi\left(g z^{\prime}\right)\right) \leq \psi\left(d\left(g z, g z^{\prime}\right)+\varphi\left(g z^{\prime}\right)\right)-\phi\left(d\left(g z, g z^{\prime}\right)+\varphi\left(g z^{\prime}\right)\right)$.

Hence, we get that $d\left(g z, g z^{\prime}\right)+\varphi\left(g z^{\prime}\right)=0$, which implies that $g z=g z^{\prime}$ and $\varphi\left(g z^{\prime}\right)=0$. Since $g$ is an injective mapping, then $z=z^{\prime}$; that is, $z$ is a unique coincidence point of $f$ and $g$. Further, if $f$ and $g$ are weakly compatible, then it is easy to show that $z$ is a unique common fixed point of $f$ and $g$. This completes the proof.

Example 11. Let $X=[0,+\infty)$ and $d(x, y)=(x-y)^{2}$. Define mappings $f, g, \varphi: X \longrightarrow X$ by

$$
f x=\frac{\left(x+x^{2}\right)}{8}, \quad g x=\frac{7\left(x+x^{2}\right)}{8}, \varphi x=x^{2}, \quad x \in[0,+\infty) .
$$

Define mappings $\psi, \phi:[0,+\infty) \longrightarrow[0,+\infty)$ with $\psi(t)=$ $t, \phi(t)=35 t / 98$.

It is clear that $f(X) \subset g(X)$. For all $x, y \in X$, we have

$$
\begin{aligned}
\psi\left(s^{p}\right. & {[d(f x, f y)+\varphi(f x)+\varphi(f y)]) } \\
& =4 \cdot\left[\left(\frac{x+x^{2}}{8}-\frac{y+y^{2}}{8}\right)^{2}+\left(\frac{x+x^{2}}{8}\right)^{2}+\left(\frac{y+y^{2}}{8}\right)^{2}\right] \\
& \leq 4 \cdot \frac{1}{64} \cdot 2\left(\left(x+x^{2}\right)^{2}+\left(y+y^{2}\right)^{2}\right)=\frac{1}{8}\left(\left(x+x^{2}\right)^{2}+\left(y+y^{2}\right)^{2}\right),
\end{aligned}
$$

$\psi(m(x, y, d, f, g, \varphi))$

$$
\begin{aligned}
\geq & \psi\left(\frac{1}{2}[d(f x, g x)+\varphi(f x)+\varphi(g x)+d(f y, g y)+\varphi(f y)+\varphi(g y)]\right) \\
= & \frac{1}{2}\left[\left(\frac{x+x^{2}}{8}-\frac{7\left(x+x^{2}\right)}{8}\right)^{2}+\left(\frac{x+x^{2}}{8}\right)^{2}+\left(\frac{7\left(x+x^{2}\right)}{8}\right)^{2}\right. \\
& \left.+\left(\frac{y+y^{2}}{8}-\frac{7\left(y+y^{2}\right)}{8}\right)^{2}+\left(\frac{y+y^{2}}{8}\right)^{2}+\left(\frac{7\left(y+y^{2}\right)}{8}\right)^{2}\right] \\
= & \frac{43}{64}\left(\left(x+x^{2}\right)^{2}+\left(y+y^{2}\right)^{2}\right),
\end{aligned}
$$




$$
\begin{aligned}
\phi(l(x, y, d, f, g, \varphi))= & \frac{35}{98} \max \{d(g x, g y)+\varphi(g x)+\varphi(g y), d(f y, g y)+\varphi(f y)+\varphi(g y)\} \\
= & \frac{35}{98} \max \left\{\left(\frac{7\left(x+x^{2}\right)}{8}-\frac{7\left(y+y^{2}\right)}{8}\right)^{2}+\left(\frac{7\left(x+x^{2}\right)}{8}\right)^{2}\right. \\
& +\left(\frac{7\left(y+y^{2}\right)}{8}\right)^{2},\left(\frac{y+y^{2}}{8}-\frac{7\left(y+y^{2}\right)}{8}\right)^{2}+\left(\frac{y+y^{2}}{8}\right)^{2} \\
& \left.+\left(\frac{7\left(y+y^{2}\right)}{8}\right)^{2}\right\} \\
\leq & \frac{35}{98} \max \left\{\frac{49}{32}\left(\left(x+x^{2}\right)^{2}+\left(y+y^{2}\right)^{2}\right), \frac{43}{32}\left(y+y^{2}\right)^{2}\right\} \\
\leq & \frac{35}{64}\left(\left(x+x^{2}\right)^{2}+\left(y+y^{2}\right)^{2}\right) .
\end{aligned}
$$

According to above inequalities, we get that

$$
\begin{aligned}
\psi\left(s^{p}[\right. & d(f x, f y)+\varphi(f x)+\varphi(f y)]) \\
\leq & \frac{1}{8}\left(\left(x+x^{2}\right)^{2}+\left(y+y^{2}\right)^{2}\right)=\frac{43}{64}\left(\left(x+x^{2}\right)^{2}+\left(y+y^{2}\right)^{2}\right) \\
& \quad-\frac{35}{64}\left(\left(x+x^{2}\right)^{2}+\left(y+y^{2}\right)^{2}\right) \\
\leq & \psi(m(x, y, d, f, g, \varphi))-\phi(l(x, y, d, f, g, \varphi)) .
\end{aligned}
$$

It follows that all conditions of Theorem 10 are satisfied with $s=2, p=2$. It is easy to obtain that 0 is the unique common fixed point of $f$ and $g$.

Note that, for $x=0, y \in(0,+\infty)$, one can calculate that

$$
\begin{aligned}
s d(f x, g y) & =\frac{49\left(y+y^{2}\right)^{2}}{32}=\max \left\{0, \frac{49\left(y+y^{2}\right)^{2}}{32}, L \frac{113\left(y+y^{2}\right)^{2}}{64}\right\} \\
& >\max \{s d(x, f x), s d(y, g y), L(d(x, g y)+d(f x, y))\} \\
& >\varphi(\max \{s d(x, f x), s d(y, g y), L(d(x, g y)+d(f x, y))\}),
\end{aligned}
$$

which implies that Theorem 1 of [10] cannot be applied to testify the existence of common fixed points of the mappings $f$ and $g$ in $X$.

If $\varphi=0$ in Theorem 10, we can get the following result:

Corollary 12. Let $(X, d)$ be a complete b-metric space with parameter $s \geq 1$, and let $f, g: X \longrightarrow X$ be given selfmappings satisfying $g$ as injective and $f(X) \subset g(X)$ where $g($ $X)$ is closed. Suppose $p \geq 2$ is a constant. If there are functions $\psi \in \Psi$ and $\phi \in \Phi$ such that

$$
\psi\left(s^{p} d(f x, f y)\right) \leq \psi\left(m_{1}(x, y, d, f, g)\right)-\phi\left(l_{1}(x, y, d, f, g)\right),
$$

where

$$
\begin{aligned}
& m_{1}(x, y, d, f, g)= \max \left\{d(g x, g y), \frac{1}{2}\{d(f x, g x)+d(f y, g y)\}, \frac{1}{2 s}\right. \\
& \cdot\{d(f x, g y)+d(f y, g x)\}\}, \\
& l_{1}(x, y, d, f, g)=\max \{d(g x, g y), d(f y, g y)\}
\end{aligned}
$$

then $f$ and $g$ have a unique coincidence point in X. Moreover, $f$ and $g$ have a unique common fixed point provided that $f$ and $g$ are weakly compatible.

If we consider the corresponding problem in the setting of metric space, that is, $s=1$ in Theorem 10, one can obtain the following:

Corollary 13. Let $(X, d)$ be a complete metric space, and let $f, g: X \longrightarrow X$ be given self-mappings satisfying $g$ as injective and $f(X) \subset g(X)$ where $g(X)$ is closed. Suppose $\varphi: X \longrightarrow[0$, $+\infty)$ is a lower semicontinuous function. If there are functions $\psi \in \Psi$ and $\phi \in \Phi$ such that

$$
\begin{aligned}
& \psi(d(f x, f y)+\varphi(f x)+\varphi(f y)) \\
& \quad \leq \psi\left(m_{2}(x, y, d, f, g, \varphi)\right)-\phi(l(x, y, d, f, g, \varphi)),
\end{aligned}
$$

where

$$
\begin{aligned}
m_{2}(x, y, d, f, g, \varphi)= & \max \left\{d(g x, g y)+\varphi(g x)+\varphi(g y), \frac{1}{2}\{d(f x, g x)\right. \\
& +\varphi(f x)+\varphi(g x)+d(f y, g y)+\varphi(f y)+\varphi(g y)\}, \frac{1}{2} \\
& \cdot\{d(f x, g y)+\varphi(f x)+\varphi(g y)+d(f y, g x)+\varphi(f y) \\
& +\varphi(g x)\}\}
\end{aligned}
$$

and $l(x, y, d, f, g, \varphi)$ is the same as Theorem 10 , then $f$ and $g$ have a unique coincidence point in X. Moreover, $f$ and $g$ have a unique common fixed point provided that $f$ and $g$ are weakly compatible.

Theorem 14. Let $(X, d)$ be a complete b-metric space with parameter $s \geq 1$ and let $f, g: X \longrightarrow X$ be given self-mappings, and one off and $g$ is continuous. Suppose $\varphi: X \longrightarrow[0,+\infty)$ is a lower semicontinuous function and $p \geq 3,0<\lambda \leq 1 / 4$ are two constants. If there are functions $\psi \in \Psi$ and $\phi \in \Phi$ such that

$$
\begin{aligned}
& \psi\left(s^{p}[d(f x, g y)+\varphi(f x)+\varphi(g y)]\right) \\
& \quad \leq \psi(n(x, y, d, f, g, \varphi))-\phi(r(x, y, d, g, \varphi)),
\end{aligned}
$$

where

$$
\begin{aligned}
& n(x, y, d, f, g, \varphi)= \lambda \max \{d(x, y)+\varphi(x)+\varphi(y), d(f x, x)+\varphi(f x) \\
&+\varphi(x)+d(y, g y)+\varphi(y)+\varphi(g y), \frac{1}{s}\{d(f x, y) \\
&+\varphi(f x)+\varphi(y)+d(x, g y)+\varphi(x)+\varphi(g y)\}\}, \\
& r(x, y, d, g, \varphi)=\max \{d(x, y)+\varphi(x)+\varphi(y), d(y, g y)+\varphi(y)+\varphi(g y)\},
\end{aligned}
$$

then $f$ and $g$ have a unique common fixed point in $X$.

Proof. Let $x_{0} \in X$ be an arbitrary point. Define a sequence \{ $\left.x_{n}\right\}$ in $X$ by $x_{2 i+1}=f x_{2 i}, x_{2 i+2}=g x_{2 i+1}$ for $i=0,1,2, \cdots$. Firstly, we prove that $f$ and $g$ have at most one common fixed point. 
Suppose that $v$ and $w$ are two different common fixed points; then, $f(v)=v \neq w=g(w)$. It follows that $d(f(v), g(w))=d($ $v, w)>0$. Applying (60) with $x=v$ and $y=w$, we obtain

$$
\begin{aligned}
& \psi(d(v, w)+\varphi(v)+\varphi(w)) \\
& \quad \leq \psi\left(s^{p}[d(f v, g w)+\varphi(f v)+\varphi(g w)]\right) \\
& \quad \leq \psi(n(v, w, d, f, g, \varphi))-\phi(r(v, w, d, g, \varphi)),
\end{aligned}
$$

where

$$
\begin{aligned}
n(v, w, d, f, g, \varphi)= & \lambda \max \{d(v, w)+\varphi(v)+\varphi(w), d(f v, v)+\varphi(f v) \\
& +\varphi(v)+d(w, g w)+\varphi(w)+\varphi(g w), \frac{1}{s}\{d(f v, w) \\
& +\varphi(f v)+\varphi(w)+d(v, g w)+\varphi(v)+\varphi(g w)\}\} \\
= & \lambda \max \{d(v, w)+\varphi(v)+\varphi(w), d(v, v)+\varphi(v) \\
& +\varphi(v)+d(w, w)+\varphi(w)+\varphi(w), \frac{1}{s}\{d(v, w) \\
& +\varphi(v)+\varphi(w)+d(v, w)+\varphi(v)+\varphi(w)\}\} \\
\leq & d(v, w)+\varphi(v)+\varphi(w),
\end{aligned}
$$

$r(v, w, d, g, \varphi)=\max \{d(v, w)+\varphi(v)+\varphi(w), d(w, g w)+\varphi(w)+\varphi(g w)\}$ $=\max \{d(v, w)+\varphi(v)+\varphi(w), d(w, w)+\varphi(w)+\varphi(w)\}$ $\geq d(v, w)+\varphi(v)+\varphi(w)$.

It follows from (62) that

$$
\begin{aligned}
& \psi(d(v, w)+\varphi(v)+\varphi(w)) \\
& \quad \leq \psi(d(v, w)+\varphi(v)+\varphi(w))-\phi(d(v, w)+\varphi(v)+\varphi(w)),
\end{aligned}
$$

which implies that $d(v, w)+\varphi(v)+\varphi(w)=0$. That is, $v=w$ and $\varphi(v)=0$. Hence, the pair $(f, g)$ has at most one common fixed point.

We suppose that $d\left(x_{n}, x_{n+1}\right)>0$ for all $n \in \mathbb{N}$. If not, for some $k, x_{2 k}=x_{2 k+1}$ and from (60), we obtain

$$
\begin{aligned}
& \psi\left(d\left(x_{2 k+1}, x_{2 k+2}\right)+\varphi\left(x_{2 k+1}\right)+\varphi\left(x_{2 k+2}\right)\right) \\
& \quad \leq \psi\left(s^{p}\left[d\left(f x_{2 k}, g x_{2 k+1}\right)+\varphi\left(f x_{2 k}\right)+\varphi\left(g x_{2 k+1}\right)\right]\right) \\
& \quad \leq \psi\left(n\left(x_{2 k}, x_{2 k+1}, d, f, g, \varphi\right)\right)-\phi\left(r\left(x_{2 k}, x_{2 k+1}, d, g, \varphi\right)\right),
\end{aligned}
$$

where

$$
\begin{aligned}
n\left(x_{2 k}, x_{2 k+1}, d, f, g, \varphi\right)= & \lambda \max \left\{d\left(x_{2 k}, x_{2 k+1}\right)+\varphi\left(x_{2 k}\right)+\varphi\left(x_{2 k+1}\right), d\left(f x_{2 k}, x_{2 k}\right)\right. \\
& +\varphi\left(f x_{2 k}\right)+\varphi\left(x_{2 k}\right)+d\left(x_{2 k+1}, g x_{2 k+1}\right)+\varphi\left(x_{2 k+1}\right) \\
& +\varphi\left(g x_{2 k+1}\right), \frac{1}{s}\left\{d\left(f x_{2 k}, x_{2 k+1}\right)+\varphi\left(f x_{2 k}\right)+\varphi\left(x_{2 k+1}\right)\right. \\
& \left.\left.+d\left(x_{2 k}, g x_{2 k+1}\right)+\varphi\left(x_{2 k}\right)+\varphi\left(g x_{2 k+1}\right)\right\}\right\} \\
= & \lambda \max \left\{d\left(x_{2 k}, x_{2 k+1}\right)+\varphi\left(x_{2 k}\right)+\varphi\left(x_{2 k+1}\right), d\left(x_{2 k+1}, x_{2 k}\right)\right. \\
& +\varphi\left(x_{2 k+1}\right)+\varphi\left(x_{2 k}\right)+d\left(x_{2 k+1}, x_{2 k+2}\right)+\varphi\left(x_{2 k+1}\right) \\
& +\varphi\left(x_{2 k+2}\right), \frac{1}{s}\left\{d\left(x_{2 k+1}, x_{2 k+1}\right)+\varphi\left(x_{2 k+1}\right)+\varphi\left(x_{2 k+1}\right)\right. \\
& \left.\left.+d\left(x_{2 k}, x_{2 k+2}\right)+\varphi\left(x_{2 k}\right)+\varphi\left(x_{2 k+2}\right)\right\}\right\} \\
\leq & \frac{1}{2} \max \left\{d\left(x_{2 k+1}, x_{2 k}\right)+\varphi\left(x_{2 k+1}\right)+\varphi\left(x_{2 k}\right), d\left(x_{2 k+1}, x_{2 k+2}\right)\right. \\
& \left.+\varphi\left(x_{2 k+1}\right)+\varphi\left(x_{2 k+2}\right)\right\} \leq d\left(x_{2 k+1}, x_{2 k+2}\right)+\varphi\left(x_{2 k+1}\right)+\varphi\left(x_{2 k+2}\right),
\end{aligned}
$$

$r\left(x_{2 k}, x_{2 k+1}, d, g, \varphi\right)=\max \left\{d\left(x_{2 k}, x_{2 k+1}\right)+\varphi\left(x_{2 k}\right)+\varphi\left(x_{2 k+1}\right), d\left(x_{2 k+1}, g x_{2 k+1}\right)\right.$ $\left.+\varphi\left(x_{2 k+1}\right)+\varphi\left(g x_{2 k+1}\right)\right\}=\max \left\{d\left(x_{2 k}, x_{2 k+1}\right)+\varphi\left(x_{2 k}\right)\right.$ $\left.+\varphi\left(x_{2 k+1}\right), d\left(x_{2 k+1}, x_{2 k+2}\right)+\varphi\left(x_{2 k+1}\right)+\varphi\left(x_{2 k+2}\right)\right\}$ $\geq d\left(x_{2 k+1}, x_{2 k+2}\right)+\varphi\left(x_{2 k+1}\right)+\varphi\left(x_{2 k+2}\right)$.

By virtue of (65) and the above inequalities, we have

$$
\begin{aligned}
& \psi\left(d\left(x_{2 k+1}, x_{2 k+2}\right)+\varphi\left(x_{2 k+1}\right)+\varphi\left(x_{2 k+2}\right)\right) \\
& \quad \leq \psi\left(n\left(x_{2 k}, x_{2 k+1}, d, f, g, \varphi\right)\right)-\phi\left(r\left(x_{2 k}, x_{2 k+1}, d, g, \varphi\right)\right) \\
& \quad \leq \psi\left(d\left(x_{2 k+1}, x_{2 k+2}\right)+\varphi\left(x_{2 k+1}\right)+\varphi\left(x_{2 k+2}\right)\right) \\
& \quad-\phi\left(d\left(x_{2 k+1}, x_{2 k+2}\right)+\varphi\left(x_{2 k+1}\right)+\varphi\left(x_{2 k+2}\right)\right),
\end{aligned}
$$

which implies that $d\left(x_{2 k+1}, x_{2 k+2}\right)+\varphi\left(x_{2 k+1}\right)+\varphi\left(x_{2 k+2}\right)=0$. That is, $x_{2 k+1}=x_{2 k+2}$. Thus, $x_{2 k}$ is a common fixed point of $f$ and $g$. If $x_{2 k+1}=x_{2 k+2}$, then using the same arguments as in the case $x_{2 k}=x_{2 k+1}$, it can be shown that $x_{2 k+1}$ is a common fixed point of $f$ and $g$.

Now take $d\left(x_{n}, x_{n+1}\right)>0$ for each $n \in \mathbb{N}$. Letting $x=x_{2 n}$ , $y=x_{2 n+1}$ in (60), as the same arguments, we obtain

$$
\begin{aligned}
& \psi\left(d\left(x_{2 n+1}, x_{2 n+2}\right)+\varphi\left(x_{2 n+1}\right)+\varphi\left(x_{2 n+2}\right)\right) \\
& \quad \leq \psi\left(n\left(x_{2 n}, x_{2 n+1}, d, f, g, \varphi\right)\right)-\phi\left(r\left(x_{2 n}, x_{2 n+1}, d, g, \varphi\right)\right)
\end{aligned}
$$

where

$$
\begin{aligned}
n\left(x_{2 n}, x_{2 n+1}, d, f, g, \varphi\right)= & \lambda \max \left\{d\left(x_{2 n}, x_{2 n+1}\right)+\varphi\left(x_{2 n}\right)+\varphi\left(x_{2 n+1}\right), d\left(x_{2 n+1}, x_{2 n}\right)\right. \\
& +\varphi\left(x_{2 n+1}\right)+\varphi\left(x_{2 n}\right)+d\left(x_{2 n+1}, x_{2 n+2}\right)+\varphi\left(x_{2 n+1}\right) \\
& +\varphi\left(x_{2 n+2}\right), \frac{1}{s}\left\{d\left(x_{2 n+1}, x_{2 n+1}\right)+\varphi\left(x_{2 n+1}\right)+\varphi\left(x_{2 n+1}\right)\right. \\
& \left.\left.+d\left(x_{2 n}, x_{2 n+2}\right)+\varphi\left(x_{2 n}\right)+\varphi\left(x_{2 n+2}\right)\right\}\right\} \\
\leq & \frac{1}{2} \max \left\{d\left(x_{2 n+1}, x_{2 n}\right)+\varphi\left(x_{2 n+1}\right)+\varphi\left(x_{2 n}\right), d\left(x_{2 n+1}, x_{2 n+2}\right)\right. \\
& \left.+\varphi\left(x_{2 n+1}\right)+\varphi\left(x_{2 n+2}\right)\right\}, \\
r\left(x_{2 n}, x_{2 n+1}, d, g, \varphi\right)= & \max \left\{d\left(x_{2 n}, x_{2 n+1}\right)+\varphi\left(x_{2 n}\right)+\varphi\left(x_{2 n+1}\right), d\left(x_{2 n+1}, x_{2 n+2}\right)\right. \\
& \left.+\varphi\left(x_{2 n+1}\right)+\varphi\left(x_{2 n+2}\right)\right\} .
\end{aligned}
$$

If for some $n, d\left(x_{2 n+1}, x_{2 n+2}\right)+\varphi\left(x_{2 n+1}\right)+\varphi\left(x_{2 n+2}\right)>d($ $\left.x_{2 n}, x_{2 n+1}\right)+\varphi\left(x_{2 n}\right)+\varphi\left(x_{2 n+1}\right)$, then it follows from (68), (69), and (70) that

$$
\begin{aligned}
& \psi\left(d\left(x_{2 n+1}, x_{2 n+2}\right)+\varphi\left(x_{2 n+1}\right)+\varphi\left(x_{2 n+2}\right)\right) \\
& \leq \psi\left(d\left(x_{2 n+1}, x_{2 n+2}\right)+\varphi\left(x_{2 n+1}\right)+\varphi\left(x_{2 n+2}\right)\right) \\
& \quad-\phi\left(d\left(x_{2 n+1}, x_{2 n+2}\right)+\varphi\left(x_{2 n+1}\right)+\varphi\left(x_{2 n+2}\right)\right)
\end{aligned}
$$

which yields that $\phi\left(d\left(x_{2 n+1}, x_{2 n+2}\right)+\varphi\left(x_{2 n+1}\right)=0\right.$ or equivalently

$$
d\left(x_{2 n+1}, x_{2 n+2}\right)+\varphi\left(x_{2 n+1}\right)+\varphi\left(x_{2 n+2}\right)=0 .
$$


That is, $d\left(x_{2 n+1}, x_{2 n+2}\right)=0$, a contradiction. Hence,

$$
\begin{aligned}
& d\left(x_{2 n+1}, x_{2 n+2}\right)+\varphi\left(x_{2 n+1}\right)+\varphi\left(x_{2 n+2}\right) \\
& \quad \leq d\left(x_{2 n}, x_{2 n+1}\right)+\varphi\left(x_{2 n}\right)+\varphi\left(x_{2 n+1}\right),
\end{aligned}
$$

for all $n \in N$. By similar arguments, we get

$d\left(x_{2 n+2}, x_{2 n+3}\right)+\varphi\left(x_{2 n+2}\right)+\varphi\left(x_{2 n+3}\right) \leq d\left(x_{2 n+1}, x_{2 n+2}\right)+\varphi\left(x_{2 n+1}\right)+\varphi\left(x_{2 n+2}\right)$.

Therefore, $\left\{d\left(x_{n}, x_{n+1}\right)+\varphi\left(x_{n}\right)+\varphi\left(x_{n+1}\right)\right\}$ is a nonincreasing sequence, and there exists a $r \geq 0$ such that

$$
\lim _{n \longrightarrow+\infty} d\left(x_{n}, x_{n+1}\right)+\varphi\left(x_{n}\right)+\varphi\left(x_{n+1}\right)=r .
$$

If $r>0$, by virtue of (68), (69), (70), and (73), one can obtain that

$$
\begin{gathered}
\psi\left(d\left(x_{2 n+1}, x_{2 n+2}\right)+\varphi\left(x_{2 n+1}\right)+\varphi\left(x_{2 n+2}\right)\right) \\
\leq \psi\left(d\left(x_{2 n}, x_{2 n+1}\right)+\varphi\left(x_{2 n}\right)+\varphi\left(x_{2 n+1}\right)\right) \\
\quad-\phi\left(d\left(x_{2 n}, x_{2 n+1}\right)+\varphi\left(x_{2 n}\right)+\varphi\left(x_{2 n+1}\right) .\right.
\end{gathered}
$$

Taking the upper limit as $n \longrightarrow+\infty$ in (76), we have

$$
\begin{aligned}
& \underset{n \longrightarrow+\infty}{\limsup } \psi\left(d\left(x_{2 n+1}, x_{2 n+2}\right)+\varphi\left(x_{2 n+1}\right)+\varphi\left(x_{2 n+2}\right)\right) \\
& \leq \limsup _{n \longrightarrow+\infty} \psi\left(d\left(x_{2 n}, x_{2 n+1}\right)+\varphi\left(x_{2 n}\right)+\varphi\left(x_{2 n+1}\right)\right) \\
&-\limsup _{n \longrightarrow+\infty} \phi\left(d\left(x_{2 n}, x_{2 n+1}\right)+\varphi\left(x_{2 n}\right)+\varphi\left(x_{2 n+1}\right)\right) \\
& \leq \limsup \psi\left(d\left(x_{2 n}, x_{2 n+1}\right)+\varphi\left(x_{2 n}\right)+\varphi\left(x_{2 n+1}\right)\right) \\
& n \longrightarrow+\infty \\
&-\liminf _{n \longrightarrow+\infty} \phi\left(d\left(x_{2 n}, x_{2 n+1}\right)+\varphi\left(x_{2 n}\right)+\varphi\left(x_{2 n+1}\right)\right),
\end{aligned}
$$

which implies that $\psi(r) \leq \psi(r)-\phi(r)$, a contradiction. It follows that

$$
\lim _{n \longrightarrow+\infty} d\left(x_{n}, x_{n+1}\right)+\varphi\left(x_{n}\right)+\varphi\left(x_{n+1}\right)=0,
$$

which yields that

$$
\lim _{n \longrightarrow+\infty} d\left(x_{n}, x_{n+1}\right)=0, \lim _{n \longrightarrow+\infty} \varphi\left(x_{n}\right)=0 .
$$

Now we shall prove that $\left\{x_{n}\right\}$ is a Cauchy sequence in $X$. To do this, it is sufficient to prove that $\left\{x_{2 n}\right\}$ is Cauchy. Suppose on the contrary that $\left\{x_{2 n}\right\}$ is not Cauchy. It follows that there exists $\varepsilon>0$ for which one can find sequences $\left\{x_{2 m_{k}}\right\}$ and $\left\{x_{2 n_{k}}\right\}$ of $\left\{x_{2 n}\right\}$ satisfying $n_{k}$ as the smallest index for which $2 m_{k}>2 n_{k}>k$,

$$
\begin{gathered}
\varepsilon \leq d\left(x_{2 m_{k}}, x_{2 n_{k}}\right), \\
d\left(x_{2 m_{k}-2}, x_{2 n_{k}}\right)<\varepsilon .
\end{gathered}
$$

Using the same technique in the proof of Theorem 10, we can deduce that

$$
\begin{aligned}
& \varepsilon \leq \liminf _{k \longrightarrow+\infty} d\left(x_{2 m_{k}}, x_{2 n_{k}}\right) \leq \limsup _{k \longrightarrow+\infty} d\left(x_{2 m_{k}}, x_{2 n_{k}}\right) \leq s \varepsilon, \\
& \frac{\varepsilon}{s} \leq \liminf _{k \longrightarrow+\infty} d\left(x_{2 m_{k}-1}, x_{2 n_{k}}\right) \leq \limsup _{k \longrightarrow+\infty} d\left(x_{2 m_{k}-1}, x_{2 n_{k}}\right) \leq s^{2} \varepsilon,
\end{aligned}
$$

$\frac{\varepsilon}{s^{2}} \leq \liminf _{k \longrightarrow+\infty} d\left(x_{2 m_{k}-1}, x_{2 n_{k}+1}\right) \leq \limsup _{k \longrightarrow+\infty} d\left(x_{2 m_{k}-1}, x_{2 n_{k}+1}\right) \leq s^{3} \varepsilon$,

$$
\frac{\varepsilon}{s} \leq \liminf _{k \longrightarrow+\infty} d\left(x_{2 m_{k}}, x_{2 n_{k}+1}\right) \leq \limsup _{k \longrightarrow+\infty} d\left(x_{2 m_{k}}, x_{2 n_{k}+1}\right) .
$$

Letting $x=x_{2 n_{k}}$ and $y=x_{2 m_{k}-1}$ in (60), we obtain

$$
\begin{aligned}
& \psi\left(d\left(x_{2 n_{k}+1}, x_{2 m_{k}}\right)+\varphi\left(x_{2 n_{k}+1}\right)+\varphi\left(x_{2 m_{k}}\right)\right) \\
& \quad \leq \psi\left(s^{p}\left[d\left(f x_{2 n_{k}}, g x_{2 m_{k}-1}\right)+\varphi\left(f x_{2 n_{k}}\right)+\varphi\left(g x_{2 m_{k}-1}\right)\right]\right) \\
& \quad \leq \psi\left(n\left(x_{2 n_{k}}, x_{2 m_{k}-1}, d, f, g, \varphi\right)\right)-\phi\left(r\left(x_{2 n_{k}}, x_{2 m_{k}-1}, d, g, \varphi\right)\right) .
\end{aligned}
$$

Here,

$$
\begin{aligned}
n\left(x_{2 n_{k}}, x_{2 m_{k}-1}, d, f, g, \varphi\right)= & \lambda \max \left\{d\left(x_{2 n_{k}}, x_{2 m_{k}-1}\right)+\varphi\left(x_{2 n_{k}}\right)\right. \\
& +\varphi\left(x_{2 m_{k}-1}\right), d\left(f x_{2 n_{k}}, x_{2 n_{k}}\right)+\varphi\left(f x_{2 n_{k}}\right) \\
& +\varphi\left(x_{2 n_{k}}\right)+d\left(x_{2 m_{k}-1}, g x_{2 m_{k}-1}\right) \\
& +\varphi\left(x_{2 m_{k}-1}\right)+\varphi\left(g x_{2 m_{k}-1}\right), \frac{1}{s}\left\{d\left(f x_{2 n_{k}}, x_{2 m_{k}-1}\right)\right. \\
& +\varphi\left(f x_{2 n_{k}}\right)+\varphi\left(x_{2 m_{k}-1}\right)+d\left(x_{2 n_{k}}, g x_{2 m_{k}-1}\right) \\
& \left.\left.+\varphi\left(x_{2 n_{k}}\right)+\varphi\left(g x_{2 m_{k}-1}\right)\right\}\right\} \\
= & \lambda \max \left\{d\left(x_{2 n_{k}}, x_{2 m_{k}-1}\right)+\varphi\left(x_{2 n_{k}}\right)\right. \\
& +\varphi\left(x_{2 m_{k}-1}\right), d\left(x_{2 n_{k}+1}, x_{2 n_{k}}\right)+\varphi\left(x_{2 n_{k}+1}\right) \\
& +\varphi\left(x_{2 n_{k}}\right)+d\left(x_{2 m_{k}-1}, x_{2 m_{k}}\right)+\varphi\left(x_{2 m_{k}-1}\right) \\
& +\varphi\left(x_{2 m_{k}}\right), \frac{1}{s}\left\{d\left(x_{2 n_{k}+1}, x_{2 m_{k}-1}\right)+\varphi\left(x_{2 n_{k}+1}\right)\right. \\
& \left.\left.+\varphi\left(x_{2 m_{k}-1}\right)+d\left(x_{2 n_{k}}, x_{2 m_{k}}\right)+\varphi\left(x_{2 n_{k}}\right)+\varphi\left(x_{2 m_{k}}\right)\right\}\right\},
\end{aligned}
$$

$$
\begin{aligned}
r\left(x_{2 n_{k}}, x_{2 m_{k}-1}, d, g, \varphi\right)= & \max \left\{d\left(x_{2 n_{k}}, x_{2 m_{k}-1}\right)+\varphi\left(x_{2 n_{k}}\right)+\varphi\left(x_{2 m_{k}-1}\right), d\left(x_{2 m_{k}-1}, g x_{2 m_{k}-1}\right)\right. \\
& \left.+\varphi\left(x_{2 m_{k}-1}\right)+\varphi\left(g x_{2 m_{k}-1}\right)\right\}=\max \left\{d\left(x_{2 n_{k}}, x_{2 m_{k}-1}\right)+\varphi\left(x_{2 n_{k}}\right)\right. \\
& \left.+\varphi\left(x_{2 m_{k}-1}\right), d\left(x_{2 m_{k}-1}, x_{2 m_{k}}\right)+\varphi\left(x_{2 m_{k}-1}\right)+\varphi\left(x_{2 m_{k}}\right)\right\} .
\end{aligned}
$$

It follows from (79) to (84) that

$$
\begin{aligned}
& \limsup _{k \longrightarrow+\infty} n\left(x_{2 n_{k}}, x_{2 m_{k}-1}, d, f, g, \varphi\right) \\
& \quad \leq \lambda \max \left\{s^{2} \varepsilon, 0, \frac{s^{3} \varepsilon+s \varepsilon}{s}\right\} \leq s^{2} \varepsilon,
\end{aligned}
$$

$$
s^{2} \varepsilon \geq \liminf _{k \longrightarrow+\infty} r\left(x_{2 n_{k}}, x_{2 m_{k}-1}, d, g, \varphi\right) \geq \frac{\varepsilon}{s} .
$$


By virtue of (85), (87), and (88), we have

$$
\begin{aligned}
\psi\left(s^{2} \varepsilon\right) & =\psi\left(s^{3} \frac{\varepsilon}{s}\right) \leq \psi\left(s^{p} \limsup _{k \rightarrow+\infty}\left[d\left(x_{2 n_{k}+1}, x_{2 m_{k}}\right)+\varphi\left(x_{2 n_{k}+1}\right)+\varphi\left(x_{2 m_{k}}\right)\right]\right) \\
& \leq \psi\left(s^{2} \varepsilon\right)-\liminf _{k \rightarrow+\infty} \phi\left(r\left(x_{2 n_{k}}, x_{2 m_{k}-1}, d, g, \varphi\right)\right),
\end{aligned}
$$

which implies that

$$
\liminf _{k \longrightarrow+\infty} r\left(x_{2 n_{k}}, x_{2 m_{k}-1}, d, g, \varphi\right)=0,
$$

a contradiction to (88). Hence, $\left\{x_{n}\right\}$ is a Cauchy sequence. The completeness of $X$ ensures that there exists a $x^{*}$ in $X$ such that

$$
\lim _{n \longrightarrow+\infty} f x_{2 n}=\lim _{n \longrightarrow+\infty} g x_{2 n+1}=x^{*} .
$$

By the definition of $\varphi$, we deduce that

$$
\varphi\left(x^{*}\right) \leq \liminf _{n \longrightarrow+\infty} \varphi\left(x_{n}\right)=0 .
$$

Now we will show that if one of the mappings $f$ and $g$ is continuous, then $f x^{*}=g x^{*}=x^{*}$. Without loss of generality, we can suppose that $f$ is continuous. It follows from (91) that

$$
x^{*}=\lim _{n \longrightarrow+\infty} f x_{2 n}=f\left(\lim _{n \longrightarrow+\infty} x_{2 n}\right)=f\left(x^{*}\right) .
$$

That is, $x^{*}$ is a fixed point of $f$.

From the contractive conditions (60), we get

$$
\begin{aligned}
& \psi\left(d\left(x^{*}, g x^{*}\right)+\varphi\left(x^{*}\right)+\varphi\left(g x^{*}\right)\right) \\
& \quad \leq \psi\left(s^{p}\left[d\left(f x^{*}, g x^{*}\right)+\varphi\left(f x^{*}\right)+\varphi\left(g x^{*}\right)\right]\right) \\
& \quad \leq \psi\left(n\left(x^{*}, x^{*}, d, f, g, \varphi\right)\right)-\phi\left(r\left(x^{*}, x^{*}, d, g, \varphi\right)\right),
\end{aligned}
$$

where

$$
\begin{aligned}
& n\left(x^{*}, x^{*}, d, f, g, \varphi\right)= \lambda \max \left\{d\left(x^{*}, x^{*}\right)+\varphi\left(x^{*}\right)+\varphi\left(x^{*}\right), d\left(f x^{*}, x^{*}\right)\right. \\
&+\varphi\left(f x^{*}\right)+\varphi\left(x^{*}\right)+d\left(x^{*}, g x^{*}\right)+\varphi\left(x^{*}\right) \\
&+\varphi\left(g x^{*}\right), \frac{1}{s}\left\{d\left(f x^{*}, x^{*}\right)+\varphi\left(f x^{*}\right)+\varphi\left(x^{*}\right)\right. \\
&\left.\left.+d\left(x^{*}, g x^{*}\right)+\varphi\left(x^{*}\right)+\varphi\left(g x^{*}\right)\right\}\right\} \\
&= \lambda \max \left\{0, d\left(x^{*}, g x^{*}\right)+\varphi\left(g x^{*}\right), \frac{1}{s}\left\{d\left(x^{*}, g x^{*}\right)+\varphi\left(g x^{*}\right)\right\}\right\} \\
& \leq d\left(x^{*}, g x^{*}\right)+\varphi\left(g x^{*}\right), \\
& r\left(x^{*}, x^{*}, d, g, \varphi\right)= \max \left\{d\left(x^{*}, x^{*}\right)+\varphi\left(x^{*}\right)+\varphi\left(x^{*}\right), d\left(x^{*}, g x^{*}\right)+\varphi\left(x^{*}\right)+\varphi\left(g x^{*}\right)\right\} \\
& \geq d\left(x^{*}, g x^{*}\right)+\varphi\left(g x^{*}\right) .
\end{aligned}
$$

It follows from (94) that

$$
\begin{aligned}
& \psi\left(d\left(x^{*}, g x^{*}\right)+\varphi\left(x^{*}\right)+\varphi\left(g x^{*}\right)\right) \\
& \quad \leq \psi\left(d\left(x^{*}, g x^{*}\right)+\varphi\left(g x^{*}\right)\right)-\phi\left(d\left(x^{*}, g x^{*}\right)+\varphi\left(g x^{*}\right)\right),
\end{aligned}
$$

Hence, $d\left(x^{*}, g x^{*}\right)+\varphi\left(g x^{*}\right)=0$, that is, $x^{*}=g x^{*}$ and $\varphi($ $\left.g x^{*}\right)=0$. This implies that $x^{*}$ is the unique common fixed point of $f$ and $g$. This completes the proof.

Example 15. Let $X=[0,1]$ and $d(x, y)=(x-y)^{2}$. Define mappings $f, g, \varphi: X \longrightarrow X$ by

$$
f x=\left\{\begin{array}{ll}
0, & x \in\left[0, \frac{1}{2}\right] \\
\frac{x}{16}, & x \in\left(\frac{1}{2}, 1\right]
\end{array}, g x=\frac{x}{16}, \varphi x=\frac{x^{2}}{4}, \quad x \in[0,1] .\right.
$$

Define mappings $\psi, \phi:[0,+\infty) \longrightarrow[0,+\infty)$ with $\psi(t)=$ $2 t, \phi(t)=3 \cdot 256 t / 8 \cdot 1413$.

Now we consider two cases:

Case 1. $x \in[0,1 / 2]$. For $y \in X$, we have

$$
\begin{array}{r}
\psi\left(s^{p}[d(f x, g y)+\varphi(f x)+\varphi(g y)]\right)=2 \cdot 8 \cdot\left[\left(\frac{y}{16}\right)^{2}+\frac{1}{4}\left(\frac{y}{16}\right)^{2}\right] \\
=\frac{5}{64} y^{2} \leq \frac{1}{4}\left(\frac{5 x^{2}}{8}+\frac{1157 y^{2}}{2048}\right), \\
\psi(n(x, y, d, f, g, \varphi)) \geq 2 \cdot \frac{1}{4}[d(f x, x)+\varphi(f x)+\varphi(x)+d(y, g y) \\
+\varphi(y)+\varphi(g y)])=\frac{1}{2}\left[x^{2}+\frac{x^{2}}{4}+\left(y-\frac{y}{16}\right)^{2}\right. \\
\left.+\frac{y^{2}}{4}+\frac{1}{4}\left(\frac{y}{16}\right)^{2}\right]=\frac{5}{8} x^{2}+\frac{1157}{2048} y^{2},
\end{array}
$$

$$
\begin{aligned}
\phi(r(x, y, d, g, \varphi))= & \frac{3 \cdot 256}{8 \cdot 1413} \max \{d(x, y)+\varphi(x)+\varphi(y), d(y, g y) \\
& +\varphi(y)+\varphi(g y)\} \\
\leq & \frac{3 \cdot 1157}{4 \cdot 6922} \max \left\{(x-y)^{2}+\frac{x^{2}}{4}+\frac{y^{2}}{4}, \frac{1157 y^{2}}{1024}\right\} \\
\leq & \frac{3 \cdot 1157}{4 \cdot 6922}\left(2 x^{2}+2 y^{2}+\frac{x^{2}}{4}+\frac{y^{2}}{4}+\frac{1157 y^{2}}{1024}\right) \\
\leq & \frac{3 \cdot 1157}{4 \cdot 6922}\left(\frac{9}{4} x^{2}+\frac{3461 y^{2}}{1024}\right) \leq \frac{3}{4}\left(\frac{5}{8} x^{2}+\frac{1157}{2048} y^{2}\right) .
\end{aligned}
$$

It follows that

$$
\begin{aligned}
\psi\left(s^{p}[\right. & d(f x, g y)+\varphi(f x)+\varphi(g y)]) \\
\leq & \frac{1}{4}\left(\frac{5 x^{2}}{8}+\frac{1157 y^{2}}{2048}\right)=\left(\frac{5 x^{2}}{8}+\frac{1157 y^{2}}{2048}\right) \\
& -\frac{3}{4}\left(\frac{5 x^{2}}{8}+\frac{1157 y^{2}}{2048}\right) \leq \psi(n(x, y, d, f, g, \varphi)) \\
& -\phi(r(x, y, d, g, \varphi)) .
\end{aligned}
$$


Case 2. $x \in(1 / 2,1]$. For $y \in X$, one can obtain

$$
\begin{aligned}
\begin{aligned}
\psi\left(s^{p}[d(f x, g y)+\varphi(f x)+\varphi(g y)]\right) & =2 \cdot 8 \cdot\left[\left(\frac{x}{16}-\frac{y}{16}\right)^{2}+\frac{1}{4}\left(\frac{x}{16}\right)^{2}+\frac{1}{4}\left(\frac{y}{16}\right)^{2}\right] \\
& =\frac{1}{16}\left((x-y)^{2}+\frac{x^{2}+y^{2}}{128}\right) \leq \frac{1}{8}\left((x-y)^{2}+\frac{x^{2}+y^{2}}{4}\right), \\
\psi(n(x, y, d, f, g, \varphi)) & \geq 2 \cdot \frac{1}{4}[d(x, y)+\varphi(x)+\varphi(y)] \\
& =\frac{1}{2}\left((x-y)^{2}+\frac{x^{2}+y^{2}}{4}\right), \\
\phi(r(x, y, d, g, \varphi))= & \frac{3 \cdot 256}{8 \cdot 1413} \max \{d(x, y)+\varphi(x)+\varphi(y), d(y, g y)+\varphi(y)+\varphi(g y)\} \\
= & \frac{3 \cdot 256}{8 \cdot 1413} \max \left\{(x-y)^{2}+\frac{x^{2}}{4}+\frac{y^{2}}{4}, \frac{1157 y^{2}}{1024}\right\} \\
\leq & \frac{3}{8}\left((x-y)^{2}+\frac{x^{2}+y^{2}}{4}\right) .
\end{aligned}
\end{aligned}
$$

It follows that

$$
\begin{aligned}
\psi\left(s^{p}[\right. & d(f x, g y)+\varphi(f x)+\varphi(g y)]) \\
\leq & \frac{1}{8}\left((x-y)^{2}+\frac{x^{2}+y^{2}}{4}\right)=\frac{1}{2}\left((x-y)^{2}+\frac{x^{2}+y^{2}}{4}\right) \\
& -\frac{3}{8}\left((x-y)^{2}+\frac{x^{2}+y^{2}}{4}\right) \leq \psi(n(x, y, d, f, g, \varphi)) \\
& -\phi(r(x, y, d, g, \varphi)) .
\end{aligned}
$$

Therefore, all conditions of Theorem 10 are satisfied with $\lambda=1 / 4, s=2, p=3$. Theorem 10 ensures that $f$ and $g$ has a unique common fixed point. It is easy to get that 0 is the unique common fixed point of $f$ and $g$.

Note that, taking $S=T=I_{x}$ in Theorem 2.1 of [11], Roshan et al. give the existence of common fixed point for mappings $f, g$ such that

$$
d(f x, g y) \leq \frac{q}{s^{4}} \max \left\{d(x, y), d(f x, x), d(g y, y), \frac{1}{2}(d(x, f y)+d(f x, y))\right\},
$$

where $q \in(0,1)$ is a constant. For $x=0, y \in(1 / 2,1]$, it is easy to calculate that for $q<1 / 16$,

$$
\begin{aligned}
d(f x, g y) & =\frac{y^{2}}{256}>\frac{q}{16} y^{2}=\frac{q}{16} \max \left\{y^{2}, 0, \frac{225 y^{2}}{256}, \frac{y^{2}}{2 \cdot 256}+\frac{y^{2}}{2}\right\} \\
& =\frac{q}{s^{4}} \max \left\{d(x, y), d(f x, x), d(g y, y), \frac{1}{2}(d(x, f y)+d(f x, y))\right\},
\end{aligned}
$$

which implies that Theorem 2.1 of [11] cannot be applied to testify the existence of common fixed points of the mappings $f$ and $g$ in $X$.

If $\varphi=0$ in Theorem 10, we can get the following result:
Corollary 16. Let $(X, d)$ be a complete b-metric space with parameter $s \geq 1$ and let $f, g: X \longrightarrow X$ be given self-mappings, and one of $f$ and $g$ is continuous. Suppose $p \geq 3,0<\lambda \leq 1 / 4$ are two constants. If there are functions $\psi \in \Psi$ and $\phi \in \Phi$ such that

$\psi\left(s^{p} d(f x, g y)\right) \leq \psi\left(n_{1}(x, y, d, f, g, \varphi)\right)-\phi\left(r_{1}(x, y, d, g, \varphi)\right)$,

where

$$
\begin{aligned}
n_{1}(x, y, d, f, g, \varphi)= & \lambda \max \{d(x, y), d(f x, x) \\
& +d(y, g y), \frac{1}{s}\{d(f x, y)+d(x, g y)\}, \\
r_{1}(x, y, d, g, \varphi)= & \max \{d(x, y), d(y, g y)\},
\end{aligned}
$$

then $f$ and $g$ have a unique common fixed point in $X$.

If we consider the corresponding problem in the setting of metric space, that is, $s=1$ in Theorem 10, we get the following:

Corollary 17. Let $(X, d)$ be a complete metric space and let $f, g: X \longrightarrow X$ be given self-mappings, and one of $f$ and $g$ is continuous. Suppose $\varphi: X \longrightarrow[0, \infty)$ is a lower semicontinuous function and $0<\lambda \leq 1 / 4$ is a constant. If there are functions $\psi \in \Psi$ and $\phi \in \Phi$ such that

$$
\begin{aligned}
& \psi(d(f x, g y)+\varphi(f x)+\varphi(g y)) \\
& \quad \leq \psi\left(n_{2}(x, y, d, f, g, \varphi)\right)-\phi(r(x, y, d, g, \varphi)),
\end{aligned}
$$

where

$$
\begin{aligned}
n_{2}(x, y, d, f, g, \varphi)= & \lambda \max \{d(x, y)+\varphi(x)+\varphi(y), d(f x, x) \\
& +\varphi(f x)+\varphi(x)+d(y, g y)+\varphi(y) \\
& +\varphi(g y), \frac{1}{s}\{d(f x, y)+\varphi(f x)+\varphi(y) \\
& +d(x, g y)+\varphi(x)+\varphi(g y)\}\},
\end{aligned}
$$

and $r(x, y, d, g, \varphi)$ is the same as Theorem 10, then $f$ and $g$ have a unique common fixed point in $X$.

Theorem 18. Let $(X, d)$ be a complete b-metric space with parameter $s \geq 1$, and let $f: X \longrightarrow X$ be a given self-mapping and $\varphi: X \longrightarrow[0,+\infty)$ be a lower semicontinuous function with $\varphi(t)=0$ for $t \in$ Fix $(f)$. Suppose $p \geq 2$ is a constant. If there are functions $\psi \in \Psi$ and $\phi \in \Phi$ such that

$$
\begin{aligned}
& \psi\left(s^{p}[d(f x, f y)+\varphi(f x)+\varphi(f y)]\right) \\
& \quad \leq \psi(h(x, y, d, f, \varphi))-\phi(q(x, y, d, f, \varphi)),
\end{aligned}
$$


where

$$
\begin{aligned}
h(x, y, d, f, \varphi)= & \max \{d(x, y)+\varphi(x)+\varphi(y), d(x, f x)+\varphi(x) \\
& +\varphi(f x), d(y, f y)+\varphi(y)+\varphi(f y), \frac{1}{2 s}\{d(x, f y) \\
& +\varphi(x)+\varphi(f y)+d(y, f x)+\varphi(y)+\varphi(f x)\}\},
\end{aligned}
$$

$q(x, y, d, f, \varphi)=\max \{d(x, y)+\varphi(x)+\varphi(y), d(y, f y)+\varphi(y)+\varphi(f y)\}$,

then $f$ has a unique fixed point $z \in X$.

Proof. The proof is similar to that of Theorem 10, so we omit it.

\section{Remark 19.}

(i) If $s=1$ in Theorem 18, then we get Theorem 2 .

(ii) If $s=1$ and $\varphi=0$ in Theorem 18 , then we get Theorem 1 . result:

According to Theorem 18, we can obtain the following

Corollary 20. Let $(X, d)$ be a complete b-metric space with parameter $s \geq 1$, and let $f: X \longrightarrow X$ be a given self-mapping and $\varphi: X \longrightarrow[0,+\infty)$ be a lower semicontinuous function with $\varphi(t)=0$ for $t \in$ Fix $(f)$. Suppose $p \geq 2$ is a constant. If there are functions $\psi \in \Psi$ and $\phi \in \Phi$ such that

$$
\begin{aligned}
& \psi\left(s^{p}[d(f x, f y)+\varphi(f x)+\varphi(f y)]\right) \\
& \quad \leq \psi(h(x, y, d, f, \varphi))-\phi(h(x, y, d, f, \varphi)),
\end{aligned}
$$

where $h(x, y, d, f, \varphi)$ is the same as Theorem 18, then $f$ has a unique fixed point $z \in X$.

\section{Application}

It is well known that an automobile suspension system is the realistic application for the spring mass system in engineering problems. Consider the motion of a spring of a car when it moves along a rough and pitted road, where the forcing term is the rough road and shock absorbers provide the damping. The external forces under which the system operates may be gravity, ground vibrations, earthquake, tension force, etc. Let $m$ be the mass of the spring and $F$ be the external force acting on it; then, the critical damped motion of this system subjected to the external force $F$ is governed by the following initial value problem:

$$
\left\{\begin{array}{l}
m \frac{d^{2} x}{d t^{2}}+l \frac{d x}{d t}-m F(t, x(t))=0 \\
x(0)=0 \\
x^{\prime}(0)=0
\end{array}\right.
$$

where $l>0$ is the damping constant and $F:[0, T] \times \mathbb{R}^{+}$ $\longrightarrow \mathbb{R}$ is a continuous function.

It is easy to show that the problem (111) is equivalent to the integral equation:

$$
x(t)=\int_{0}^{T} \gamma(t, r) F(r, x(r)) d r, \quad t \in[0, T]
$$

where $\gamma(t, r)$ is Green's function given by

$$
\gamma(t, r)=\left\{\begin{array}{ll}
\frac{1-e^{\mu(t-r)}}{\mu}, & 0 \leq r \leq t \leq T \\
0, & 0 \leq t \leq r \leq T
\end{array},\right.
$$

where $\mu=l / m$ is a constant.

In this section, by using Corollary 20, we will show the existence of a solution to the integral equation:

$$
x(t)=\int_{0}^{T} G(t, r, x(r)) d r .
$$

Let $X=C([0, T])$ be the set of real continuous functions defined on $[0, T]$. For $p \geq 1$, we define

$$
d(x, y)=(\rho(x, y))^{p}=\sup _{t \in[0, T]}|x(t)-y(t)|^{p} \quad \text { for all } x, y \in X .
$$

It is easy to prove that $(X, d)$ is a complete $b$-metric space with $s=2^{p-1}$.

Consider the mapping $f: X \longrightarrow X$ defined by

$$
f x(t)=\int_{0}^{T} G(t, r, x(r)) d r
$$

Theorem 21. Consider equation (114) and suppose that

(i) $G:[0, T] \times[0, T] \times \mathbb{R} \longrightarrow \mathbb{R}^{+}$is continuous

(ii) there exists a continuous function $\gamma:[0, T] \times[0, T]$ $\longrightarrow \mathbb{R}^{+}$such that

$$
\sup _{t \in[0, T]} \int_{0}^{T} \gamma(t, r) d r \leq 1
$$

(iii) there exists a constant $L \in(0,1)$ such that for $(t, r)$ $\in[0, T] \times[0, T]$,

$$
|G(t, r, x(r))-G(t, r, y(r))| \leq \sqrt[p]{\frac{1-L}{s^{p}}} \gamma(t, r)|x(r)-y(r)| .
$$

Then, the integral equation (114) has a unique solution $x \in X$ 
Proof. For $x, y \in X$, by virtue of assumptions (i)-(iii), we have

$$
\begin{aligned}
s^{p} d(f x(t), f y(t)) & =s^{p} \sup _{t \in[0, T]}|f x(t)-f y(t)|^{p} \\
& =s^{p} \sup _{t \in[0, T]}\left|\int_{0}^{T} G(t, r, x(r)) d r-\int_{0}^{T} G(t, r, y(r)) d r\right|^{p} \\
& \leq s^{p} \sup _{t \in[0, T]}\left(\int_{0}^{T}|G(t, r, x(r))-G(t, r, y(r))| d r\right)^{p} \\
& \leq s^{p} \sup _{t \in[0, T]}\left(\int_{0}^{T} \sqrt[p]{\frac{1-L}{s^{p}}} \gamma(t, r)|x(r)-y(r)| d r\right)^{p} \\
& \leq s^{p} \sup _{t \in[0, T]}\left(\int_{0}^{T} \sqrt[p]{\frac{1-L}{s^{p}}} \gamma(t, r) d r\right)^{p} \sup _{t \in[0, T]}|x(t)-y(t)|^{p} \\
& \leq(1-L) h(x(t), y(t), d, f, \varphi),
\end{aligned}
$$

which implies that

$$
s^{p} d(f x(t), f y(t)) \leq(1-L) h(x(t), y(t), d, f, \varphi) .
$$

Therefore, letting $\psi(t)=t, \phi(t)=L t$, and $\varphi(t)=0$, all the conditions of Corollary 20 are satisfied. As a result, the mapping $f$ has a unique fixed point $x \in X$, which is a solution of the integral equation (114).

Remark 22. If we let $G(t, r, x(r))=\gamma(t, r) F(r, x(r)), \mid F(r, x($ $r))-F(r, y(r))\left|\leq \sqrt[p]{(1-L) / s^{p}}\right| x(r)-y(r) \mid$ and $\mu, T$ satisfy $\mu \geq T$, then all the conditions of Theorem 21 are satisfied, which implies that the problem (111) has a unique solution.

\section{Conclusions}

In this manuscript, we introduced a new class of generalized weakly contractive mappings and established common fixed point results involving this new class of mappings in the framework of $b$-metric spaces. Further, we provided examples that elaborated the useability of our results. Meanwhile, we presented an application to the existence of solutions to an integral equation by means of one of our results.

\section{Data Availability}

No data were used to support this study.

\section{Conflicts of Interest}

The authors declare that they have no conflicts of interest regarding the publication of this paper.

\section{Authors' Contributions}

All authors contributed equally and significantly in writing this article. All authors read and approved the final manuscript.

\section{Acknowledgments}

This work was financially supported by the Science and Research Project Foundation of the Education Department of Liaoning Province (Nos LQN201902 and LJC202003).

\section{References}

[1] S. Banach, "Surles operations dans ensembles abstraits et leur application aux equations integrales," Fundamenta Mathematicae, vol. 3, no. 3, pp. 51-57, 1922.

[2] S. Czerwik, "Contraction mappings in b-metric spaces," Acta Mathematica et Informatica Universitatis Ostraviensis, vol. 1, pp. 5-11, 1993.

[3] H. Aydi, M. Bota, and S. Moradi, "A common fixed points for weak $\phi$-contractions on $b$-metric spaces," Fixed Point Theory, vol. 13, pp. 337-346, 2012.

[4] V. Berinde, "Generalized contractions in quasimetric spaces," Seminar on Fixed Point Theory Preprint, vol. 3, pp. 3-9, 1993.

[5] M. Pacurar, "A fixed point result for $\phi$-contractions and fixed points on $b$-metric spaces without the boundness assumption," Polytechnica Posnaniensis, vol. 43, pp. 127-136, 2010.

[6] M. B. Zada, M. Sarwar, and P. Kumam, "Fixed point results for rational type contraction in $b$-metric spaces," International Journal of Analysis and Applications, vol. 16, no. 6, pp. 904920, 2018.

[7] S. Hussain, M. Sarwar, and C. Tunc, "Periodic fixed point theorems via rational type contraction in $b$-metric spaces," Journal of Mathematical Analysis, vol. 10, no. 3, pp. 61-67, 2019.

[8] M. B. Zada, M. Sarwar, F. Jarad, and T. Abdeljawad, "Common fixed point theorem via cyclic $(\alpha, \beta)-(\psi, \phi)$ s-contraction with applications," Symmetry, vol. 11, no. 2, p. 15, 2019.

[9] S. Hussain, M. Sarwar, and Y. Li, " $n$-tupled fixed point results with rational type contraction in $b$-metric spaces," European Journal of Pure and Applied Mathematics, vol. 11, no. 1, pp. 331-351, 2018.

[10] W. Shatanawi, A. Pitea, and R. Lazovic, "Contraction conditions using comparison functions on $b$-metric spaces," Fixed Point Theory and Applications, vol. 2014, Article ID 135, 2014.

[11] M. Abbas, J. R. Roshan, and S. Sedghi, "Common fixed point of four maps in b-metric spaces," Hacettepe Journal of Mathematics and Statistics, vol. 43, no. 4, pp. 613-624, 2014.

[12] M. Iqbal, A. Batool, O. Ege, and M. Sen, "Fixed point of almost contraction in b-metric spaces," Journal of Mathematics, vol. 2020, Article ID 3218134, 6 pages, 2020.

[13] N. Hussain and M. Shah, "KKM mappings in cone $b$-metric spaces," Computers \& Mathematcs with Applications, vol. 61, pp. 1677-1684, 2011.

[14] M. Younis, D. Singh, S. Radenovic, and M. Imdad, "Convergence theorems for generalized contractions and applications," Filomat, vol. 34, no. 3, pp. 945-964, 2020.

[15] H. Aydi, "Fixed point results for weakly contractive mappings in ordered partial metric spaces," Journal of Advanced Mathematical Studies, vol. 4, pp. 1-12, 2011.

[16] B. Samet, C. Vetro, and P. Vetro, "Fixed point theorems for $\alpha-\psi$-contractive type mappings," Nonlinear Analysis: Theory, Methods \& Applications, vol. 75, no. 4, pp. 2154-2165, 2012.

[17] K. Zoto and I. Vardhami, "Common fixed point results for generalized $\alpha_{s^{p}}$ contractive mappings and applications," Journal of Function Spaces, vol. 2018, Article ID 1282414, 11 pages, 2018. 
[18] K. Zoto, B. E. Rhoades, and S. Radenovic, "Some generalizations for $(\alpha-\psi, \phi)$-contractions in $b$-metric-like spaces and an application," Fixed Point Theory and Applications, vol. 2017, no. 1, 2017.

[19] H. Isik, B. Mohammadi, V. Parvaneh, and C. Park, "Extended quasi $b$-metric-like spaces and some fixed point theorems for contractive mappings," Applied Mathematics E-Notes, vol. 20, pp. 204-214, 2020.

[20] H. M. Abu-Donia, H. A. Atia, and O. M. A. Khater, "Fixed point theorem for compatible mappings in intuitionistic fuzzy 3-metric spaces," Thermal Science, vol. 24, pp. S371-S376, 2020.

[21] O. Ege, "Complex valued rectangular b-metric spaces and an application to linear equations," Journal of Nonlinear Sciences and Applications, vol. 8, no. 6, pp. 1014-1021, 2015.

[22] M. Younis, N. Fabiano, Z. Fadail, Z. Mitrovic, and S. Radenovic, "Some new observations on fixed point results in rectangular metric spaces with applications to chemical sciences," Military Technical Courier, vol. 69, p. 23, 2021.

[23] Z. D. Mitrovic, H. Isik, and S. Radenovic, "The new results in extended $b$-metric spaces and applications," International Journal of Nonlinear Analysis and Applications, vol. 11, no. 1, pp. 473-482, 2020.

[24] R. Miculescu and A. Mihail, "New fixed point theorems for setvalued contractions in b-metric spaces," Journal of Fixed Point Theory and Applications, vol. 19, no. 3, pp. 2153-2163, 2017.

[25] T. Suzuki, "Basic inequality on a $b$-metric space and its applications," Journal of Inequalities and Applications, vol. 2017, no. 1, 2017.

[26] M. Younis, D. Singh, and A. Petrusel, "Applications of graph Kannan mappings to the damped spring-mass system and deformation of an elastic beam," Discrete Dynamics in Nature and Society, vol. 2019, Article ID 1315387, 9 pages, 2019.

[27] M. Younis, D. Singh, M. Asadi, and V. Joshi, "Results on contractions of Reich type in graphical $b$-metric spaces with applications," Filomat, vol. 33, no. 17, pp. 5723-5735, 2019.

[28] A. Gholidahneh, S. Sedghi, O. Ege, Z. D. Mitrovic, and M. de la Sen, "The Meir-Keeler type contractions in extended modular $b$-metric spaces with an application," AIMS Math, vol. 6, no. 2, pp. 1781-1799, 2021.

[29] Y. Alber and S. Guerre-Delabriere, "Principles of weakly contractive maps in Hilbert spaces," in New Results in Operator Theory and Its Applications. Operator Theory: Advances and Applications, I. Gohberg and Y. Lyubich, Eds., vol. 98, Birkhauser, Basel, Switzerland, 1997.

[30] B. E. Rhoades, "Some theorems on weakly contractive maps," Nonlinear Analysis, vol. 47, no. 4, pp. 2683-2693, 2001.

[31] T. Abdeljawad, "Fixed points for generalized weakly contractive mappings in partial metric spaces," Mathematical and Computer Modelling, vol. 54, no. 11-12, pp. 2923-2927, 2011.

[32] H. Aydi, "On common fixed point theorems for $(\psi, \varphi)$-generalized $f$-weakly contractive mappings," Miskolc Mathematical Notes, vol. 14, pp. 19-30, 2013.

[33] H. Aydi, E. Karapinar, and W. Shatanawi, "Coupled fixed point results for $(\psi, \varphi)$-weakly contractive condition in ordered partial metric spaces," Computers \& Mathematcs with Applications, vol. 62, pp. 4449-4460, 2011.

[34] K. Chi, E. Karapinar, and T. D. Thanh, "On the fixed point theorems for generalized weakly contractive mappings on partial metric spaces," The Bulletin of the Iranian Mathematical Society, vol. 30, pp. 369-381, 2013.
[35] S. Cho, "Fixed point theorems for weakly $\alpha$-contractive mappings with application," Applied Mathematical Sciences, vol. 7, pp. 2953-2965, 2013.

[36] B. Choudhury, P. Konar, B. E. Rhoades, and N. Metiya, "Fixed point theorems for generalized weakly contractive mappings," Nonlinear Analysis, vol. 74, pp. 2116-2126, 2011.

[37] D. Doric, "Common fixed point for generalized $(\psi, \varphi)$-weak contractions," Applied Mathematics Letters, vol. 22, pp. 1896-1900, 2009.

[38] P. Dutta and B. S. Choudhury, "A generalization of contraction principle in metric spaces," Fixed Point Theory and Applications, vol. 2008, 2008.

[39] H. Isik and D. Turkoglu, "Fixed point theorems for weakly contractive mappings in partially ordered metric-like spaces," Fixed Point Theory and Applications, vol. 2013, no. 1, 2013.

[40] H. Lakzian and B. Samet, "Fixed points for $(\psi, \varphi)$-weakly contractive mappings in generalized metric spaces," Applied Mathematics Letters, vol. 25, pp. 902-906, 2012.

[41] S. Moradi and A. Farajzadeh, "On fixed point of $(\psi-\varphi)$-weak and generalized $(\psi-\varphi)$-weak contraction mappings," Applied Mathematics Letters, vol. 25, pp. 1257-1262, 2012.

[42] O. Popescu, "Fixed points for $(\psi, \varphi)$-weak contractions," Applied Mathematics Letters, vol. 24, pp. 1-4, 2011.

[43] N. Jamal, T. Abdeljawad, M. Sarwar, N. Mlaiki, and P. S. Kumari, "Some valid generalizations of Boyd and Wong inequality and $(\psi, \phi)$-weak contraction in partially ordered $b$ -metric spaces," International Journal of Mathematics and Mathematical Sciences, vol. 2020, Article ID 9307302, 13 pages, 2020.

[44] S. Cho, "Fixed point theorems for generalized weakly contractive mappings in metric spaces with applications," Fixed Point Theory and Applications, vol. 2018, no. 1, 2018.

[45] S. L. Singh and B. Prasad, "Some coincidence theorems and stability of iterative procedures," Computers \& Mathematcs with Applications, vol. 55, no. 11, pp. 2512-2520, 2008.

[46] A. Aghaiani, M. Abbas, and J. R. Roshan, "Common fixed point of generalized weak contractive mappings in partially ordered b-metric spaces," Mathematica Slovaca, vol. 2014, pp. 941-960, 2014.

[47] G. Jungck, "Compatible mappings and common fixed points," International Journal of Mathematical Sciences, vol. 9, pp. 771-779, 1986. 